\title{
XMM-Newton study of the lensing cluster of galaxies CL 0024+17 $7^{\star}, \star$
}

\author{
Y.-Y. Zhang ${ }^{1}$, H. Böhringer ${ }^{1}$, Y. Mellier ${ }^{2}$, G. Soucail ${ }^{3}$, and W. Forman ${ }^{4}$ \\ ${ }^{1}$ Max-Planck-Institut für extraterrestrische Physik, Giessenbachstraße, 85748 Garching, Germany \\ e-mail: yyzhang@mpe.mpg.de \\ 2 Institut d'Astrophysique de Paris, 98bis Bd. Arago, 75014 Paris, France \\ 3 Observatoire Midi-Pyrénées, Laboratoire d'Astrophysique, MR 5572, 14 avenue E. Belin, 31400 Toulouse, France \\ ${ }^{4}$ Harvard-Smithsonian Center for Astrophysics (CFA), 60 Garden Street, Cambridge, MA 02138, USA
}

Received 14 May 2004 / Accepted 27 August 2004

\begin{abstract}
We present a detailed gravitational mass measurement based on the XMM-Newton imaging spectroscopy analysis of the lensing cluster of galaxies CL $0024+17$ at $z=0.395$. The emission appears approximately symmetric. However, on the scale of $r \sim 3.3^{\prime}$ some indication of elongation is visible in the northwest-southeast (NW-SE) direction from the hardness ratio map (HRM). Within $3^{\prime}$, we measure a global gas temperature of $3.52 \pm 0.17 \mathrm{keV}$, metallicity of $0.22 \pm 0.07$, and bolometric luminosity of $2.9 \pm 0.1 \times 10^{44} h_{70}^{-2} \mathrm{erg} \mathrm{s}^{-1}$. We derive a temperature distribution with an isothermal temperature of $3.9 \mathrm{keV}$ to a radius of $1.5^{\prime}$ and a temperature gradient in the outskirts $\left(1.3^{\prime}<r<3^{\prime}\right)$. Under the assumption of hydrostatic equilibrium, we measure gravitational mass and gas mass fraction to be $M_{200}=2.0 \pm 0.3 \times 10^{14} h_{70}^{-1} M_{\odot}$ and $f_{\text {gas }}=0.20 \pm 0.03 h_{70}^{-3 / 2}$ at $r_{200}=$ $1.05 h_{70}^{-1} \mathrm{Mpc}$ using the observed temperature profile. The complex structure in the core region is the key to explaining the discrepancy in gravitational mass determined from XMM-Newton X-ray observations and HST optical lensing measurements.
\end{abstract}

Key words. galaxies: clusters: individual: CL 0024+17 - cosmology: dark matter - gravitational lensing cosmology: observations $-\mathrm{X}$-rays: galaxies: clusters

\section{Introduction}

One of the optically most prominent, but also most puzzling distant lensing galaxy clusters, is CL $0024+17$ at a redshift of $z=0.395$ (Gunn \& Oke 1975). Its high galaxy density led to the early discovery by Humason \& Sandage (1957), and the cluster has since been the subject of many studies. It was one of the first clusters to display the so-called Butcher-Oemler effect (Butcher \& Oemler 1978; Dressler \& Gunn 1982; Dressler et al. 1985), and Schneider et al. (1986) described it as a very rich optical cluster.

Koo (1988) discovered gravitational arcs in CL 0024+17, and it was subsequently studied extensively (Mellier et al. 1991; Kassiola et al. 1992, 1994; Wallington et al. 1995; Colley et al. 1996; Smail et al. 1997; Tyson et al. 1998; Broadhurst et al. 2000; Shapiro \& Iliev 2000; Treu et al. 2003; Kneib et al. 2003). A total of eight arc-like lensed images from the same background galaxy were identified (e.g. Tyson et al. 1998). The redshift of the lensed galaxy was determined by

^ This work is based on observations made with the XMM-Newton, an ESA science mission with instruments and contributions directly funded by ESA member states and the USA (NASA).

$\star \star$ Based on observations made with the European Southern Observatory telescopes obtained from the ESO/ST-ECF Science Archive Facility.
Broadhurst et al. (2000) to be $z=1.675$. Bonnet et al. (1994) detected a weak shear signal in this cluster out to a radius of $2.1 h_{70}^{-1} \mathrm{Mpc}$ and inferred a high gravitational mass of about 1.4-2.9 $\times 10^{15} h_{70}^{-1} M_{\odot}$. Recently Kneib et al. (2003) revised this lensing mass estimate downward on the basis of a very detailed Hubble Space Telescope (HST) mapping of the cluster which yielded a gravitational mass of $M_{200}=5.7_{-1.0}^{+1.1} \times$ $10^{14} h_{70}^{-1} M_{\odot}$ out to $r_{200}=1.7 h_{70}^{-1} \mathrm{Mpc}$. In spite of the massive appearance of CL 0024+17 at optical wavelengths, the cluster is relatively faint in X-rays with a ROSAT determined luminosity of $L_{\mathrm{X}}=1.22 \pm 0.08 \times 10^{44} h_{70}^{-2} \mathrm{erg} \mathrm{s}^{-1}$ in the $0.1-2.4 \mathrm{keV}$ band (Böhringer et al. 2000), an ASCA determined temperature of $5.7_{-2.1}^{+4.2} \mathrm{keV}$ (Soucail et al. 2000), and a Chandra temperature of $4.47_{-0.27}^{+0.42} \mathrm{keV}$ (Ota et al. $2004 ; M_{200}=4.6_{-0.5}^{+0.7} \times$ $10^{14} h_{70}^{-1} M_{\odot}$ to $\left.r_{200}=1.4 h_{70}^{-1} \mathrm{Mpc}\right)$. In addition to the surprisingly low X-ray luminosity in comparison to its optical prominence, the detailed analysis of the X-ray observations yield a total cluster mass in the range $M_{200}=2-4.6 \times 10^{14} h_{70}^{-1} M_{\odot}$ that is 1.3-3 times lower than the weak lensing determined mass (Soucail et al. 2000; Böhringer et al. 2000; Ota et al. 2004). The discrepancy is even larger at the arc radius $\sim 0.143 h_{70}^{-1} \mathrm{Mpc}$ (e.g. Broadhurst et al. 2000).

A hint of the explanation for this mass discrepancy came from the detailed analysis of the galaxy dynamics in CL 0024+17 based on 2300 redshifts of galaxy members 
(Czoske et al. 2001, 2002). Czoske et al. (2001, 2002) found that the line-of-sight velocity distribution is not that of a relaxed cluster and is at least bimodal. They also demonstrated that the redshifts can approximately be explained by a line-of-sight merger of two systems with a mass ratio of the order of 1:2. Also the weak lensing analysis shows a mass distribution with substructure, modeled as a bimodal distribution of two systems with slightly different central positions in the plane of the sky (Kneib et al. 2003). The infrared observations imply significant star forming activity with Star Formation Rates (SFRs) one to two orders of magnitude higher than those computed from the optical. The underestimation of the SFRs in the optical is due to absorption by dust (Coia et al. 2003).

Using the capability of XMM-Newton to perform imaging spectroscopy at high angular resolution and thus allowing us to study the density and temperature structure of the intracluster medium (ICM), we performed an XMM-Newton observation of CL 0024+17 to shed new light on this enigmatic system.

This paper is structured as follows. In Sect. 2, we describe a double background subtraction method, which is developed to provide precise spectral background removal. In Sect. 3, we analyse the properties of the hot gas in the galaxy cluster CL $0024+17$, then determine the total mass, projected gravitational mass, and gas mass fraction based on precise temperature and gas density profiles. In Sect. 4, we discuss the structures in the cluster and possible solutions for the discrepancy between the X-ray and HST lensing measurements in the gravitational mass. In Sect. 5, we present our conclusions. We adopt a flat $\Lambda \mathrm{CDM}$ cosmology with density parameter $\Omega_{\mathrm{m}}=0.3$ and Hubble constant $H_{0}=70 \mathrm{~km} \mathrm{~s}^{-1} \mathrm{Mpc}^{-1}$. Thus $1^{\prime}=0.320 h_{70}^{-1} \mathrm{Mpc}$. All coordinates are given in epoch $\mathbf{J} 2000$. We adopt the solar abundance values of Anders \& Grevesse (1989). Error bars correspond to the $68 \%$ confidence level, unless explicitly stated otherwise.

\section{Method}

\subsection{Data preparation}

CL 0024+17 was observed on Jan. 6th, 2001 for a total exposure of $52.1 \mathrm{ks}, 52.1 \mathrm{ks}$ and $48.3 \mathrm{ks}$ for MOS1, MOS2 and pn, respectively, by XMM-Newton with the European Photon Imaging Camera (EPIC) in standard Full Frame (FF) mode and Extended Full Frame (EFF) mode for MOS and pn, respectively. For all detectors, the thin filter was used. Data reduction and calibration was carried out using the XMM-Newton Science Analysis System (SAS5.4.1). The central position of the observation is RA $=00^{\mathrm{h}} 26^{\mathrm{m}} 35^{\mathrm{s}} .7$, Dec $=17^{\circ} 09^{\prime} 35^{\prime \prime} .8$.

Above $10 \mathrm{keV}$, there is little $\mathrm{X}$-ray emission from the cluster due to the low telescope efficiency. The particle background therefore completely dominates. The cluster emission is not variable, so any spectral range can be used for temporal variability studies of the background. The 10-12 keV (12-14 keV) energy band (binned in $100 \mathrm{~s}$ intervals) was used to monitor the particle background and to excise periods of high particle flux for MOS (pn). In this screening process we use the settings $F L A G=0$ and PATTERN $\leq 12($ PATTERN $\leq 4)$ for MOS (pn).
We reject those time intervals affected by flares in which the detector countrate (ctr) exceeds a threshold of $2 \sigma$ above the average ctr, where the average and the variance have been iteratively determined from the ctr histogram below the rejection threshold. We screened the data using the thresholds of 21.4, 22.3 and 56.2 for MOS1, MOS2 and pn. The net exposure time is $46.6 \mathrm{ks}, 46.0 \mathrm{ks}$ and $42.9 \mathrm{ks}$ for MOS1, MOS2 and pn, respectively.

\subsection{Background analysis}

We do not expect any cluster emission outside 5.4', the virial radius estimated from the redshift $(z=0.395$; Gunn \& Oke 1975) and the Chandra average temperature measurement (4.47 keV, Ota et al. 2004). Thus the cluster emission covers less than half of the field of view (FOV) of the XMM-Newton telescope detectors. Actually the significant cluster emission extends only to $3^{\prime}$ in the surface brightness of the XMM-Newton observations. Thus we use the $5<r<5.5^{\prime}$ region in detector coordinates to compare the background conditions of the XMM-Newton pointing of the Chandra Deep Field South (CDFS, which we use as background field for the spectral analysis) with that of CL 0024+17. The background of CL $0024+17$ is higher than that of the background field (CDFS) by $\sim 30 \%$ for MOS and $\sim 10 \%$ for pn on average in the complete energy band, in which the difference is mainly produced in the low energy band below $1 \mathrm{keV}$.

In the spectral analysis, the regions not affected by cluster emission enable us to study the residual background in the source observation compared to the background observation. We applied a double background subtraction method as described in Zhang et al. (2004a) using the results of the residual background modeling. In summary the spectral analysis is performed in two steps using the software package XSPEC11.3.0: (i) a power law model for the residual background (background difference) is obtained in XSPEC from a comparison of the outer region (5-5.5') of the target and background fields (cf. Table 1); (ii) the spectral modeling is performed in XSPEC with the cluster region of the target field as source data, the region in the same detector coordinates in the background field as background and the residual background as a second model component with model parameters fixed to the values found in step (i). The overall spectra were fitted by a "mekal" model (Mewe et al. 1985; Mewe et al. 1986; Kaastra 1992; Liedahl et al. 1995; Arnaud \& Rothenflug 1985; Arnaud \& Raymond 1992) with Galactic absorption (Dickey \& Lockman 1990; $n_{\mathrm{H}}=4.23 \times 10^{20} \mathrm{~cm}^{-2}$ ). The results with and without introducing a residual background are consistent within $1 \sigma$, e.g. the temperature (metallicity) of the $r<3^{\prime}$ region are $3.52 \pm 0.17$ $(0.22 \pm 0.07)$ and $3.61 \pm 0.28(0.22 \pm 0.11)$.

In the image analysis for the surface brightness we carry out a vignetting correction for CDFS and CL 0024+17, respectively. We derive the CDFS surface brightness in the same detector coordinates as CL $0024+17$ and scale it using the ctr ratio of CL $0024+17$ and CDFS in the 10-12 keV (12-14 keV) band for MOS (pn). After subtracting the scaled CDFS as a background from CL 0024+17, we use a constant model to fit the 
Table 1. Parameters of the residual background model fitted in the 0.4-15 keV band. Column (1): instrument; Cols. (2) and (3): index and normalization of the power law residual background in which the normalization was scaled to $1 \operatorname{arcmin}^{2}$ in units of $10^{-4} \mathrm{cts} \mathrm{s}^{-1} \mathrm{keV}^{-1} \operatorname{arcmin}^{-2}$.

\begin{tabular}{lcc}
\hline \hline Instrument & Index & Normalization \\
\hline MOS1 & 1.95 & 1.00 \\
MOS2 & 2.21 & 0.64 \\
pn & 1.95 & 2.25 \\
\hline
\end{tabular}

residual soft X-ray background which is quite flat in the outer region $\left(5<r<10^{\prime}\right)$.

For comparison, we also used the blank sky background from Lumb et al. (2002) and found that the parameters change less than $1 \sigma$ for both spectral and surface brightness analyses.

\subsection{Point source subtraction}

In a preliminary spectral analysis without subtracting the point sources we found contamination in both the soft and hard bands, especially for $\mathrm{pn}$.

Therefore we generated a list of the bright point sources using SAS task "edetect_chain" applied to five energy bands: 0.3-0.5 keV, 0.5-2 keV, 2-4.5 keV, 4.5-7.5 keV, 7.5-12 keV. The sources detected include fourteen of sixteen sources which were found in ROSAT HRI observations (Soucail et al. 2000) except that S14 and S15 are out of the FOV of XMM-Newton. Since we carried out analyses only in the $r<8^{\prime}$ region, only the brightest fourteen point sources within this region (including nine ROSAT HRI sources, S1, S2, S3, S4, S5, S6, S8, S9 and S10 in Soucail et al. 2000) were subtracted from the source events using a radius of $40^{\prime \prime}$, which is comparable to the XMM-Newton Point Spread Function (PSF) cutoff radius of $\sim 45^{\prime \prime}$, for each source that encloses nearly $90 \%$ of the flux of the point sources in both the spectroscopic and image analyses (cf. Table 2).

\section{Results}

\subsection{Image analysis}

The images in the $0.5-2 \mathrm{keV}$ band for MOS and pn were corrected for vignetting using their weighted exposure maps and were then combined. We created an adaptively-smoothed image from the combined image with a maximum sigma (the width of the smoothing Gaussian) of $12^{\prime \prime} \times 12^{\prime \prime}$ and a signal-to-noise of 5.0 (see Fig. 1). Superposed XMM-Newton $\mathrm{X}$-ray contours indicate an elongation in the northwestsoutheast (NW-SE) direction and substructure in the NW $\left(\mathrm{RA}=00^{\mathrm{h}} 26^{\mathrm{m}} 25^{\mathrm{s}} .8\right.$, Dec $=17^{\circ} 12^{\prime} 03^{\prime \prime} .7, \sim 3.3^{\prime}$ from the center $)$.

We found no sign of the substructure described in Bonnet et al. (1994) which is $\sim 6.9^{\prime}$ from the cluster center (RA = $00^{\mathrm{h}} 26^{\mathrm{m}} 35^{\mathrm{s}} .7$, Dec $\left.=17^{\circ} 09^{\prime} 35^{\prime \prime} .8\right)$ to the northeast $(\mathrm{NE})$. However, we observed the substructure, described in Kneib et al. (2003) and Czoske et al. (2001, 2002), located 3.3' NW from the cluster center covering $1^{\prime}$ with a temperature
Table 2. Parameters for the brightest point sources detected in the $r<8^{\prime}$ region. Column (1): No. of the point source; Cols. (2-3): central position; Col. (4): flux determined out to a radius of $20^{\prime \prime}$ in the $0.3-12 \mathrm{keV}$ band.

\begin{tabular}{cccc}
\hline \hline No. & RA & Dec & $\begin{array}{c}\text { Flux } \\
\left(10^{-13} \mathrm{cgs}\right)\end{array}$ \\
\hline 1 & 002612.8 & 170346.8 & 1.317 \\
2 & 002630.9 & 171014.9 & 0.933 \\
3 & 002700.0 & 170422.4 & 0.890 \\
4 & 002631.0 & 171654.2 & 0.760 \\
5 & 002620.1 & 171703.1 & 0.677 \\
6 & 002705.3 & 170640.6 & 0.671 \\
7 & 002644.2 & 170229.4 & 0.581 \\
8 & 002626.1 & 170937.3 & 0.558 \\
9 & 002707.5 & 170748.6 & 0.542 \\
10 & 002617.2 & 170306.1 & 0.400 \\
11 & 002645.8 & 171230.5 & 0.391 \\
12 & 002617.9 & 170945.8 & 0.259 \\
13 & 002703.7 & 170721.5 & 0.224 \\
14 & 002642.8 & 170830.9 & 0.060 \\
\hline
\end{tabular}

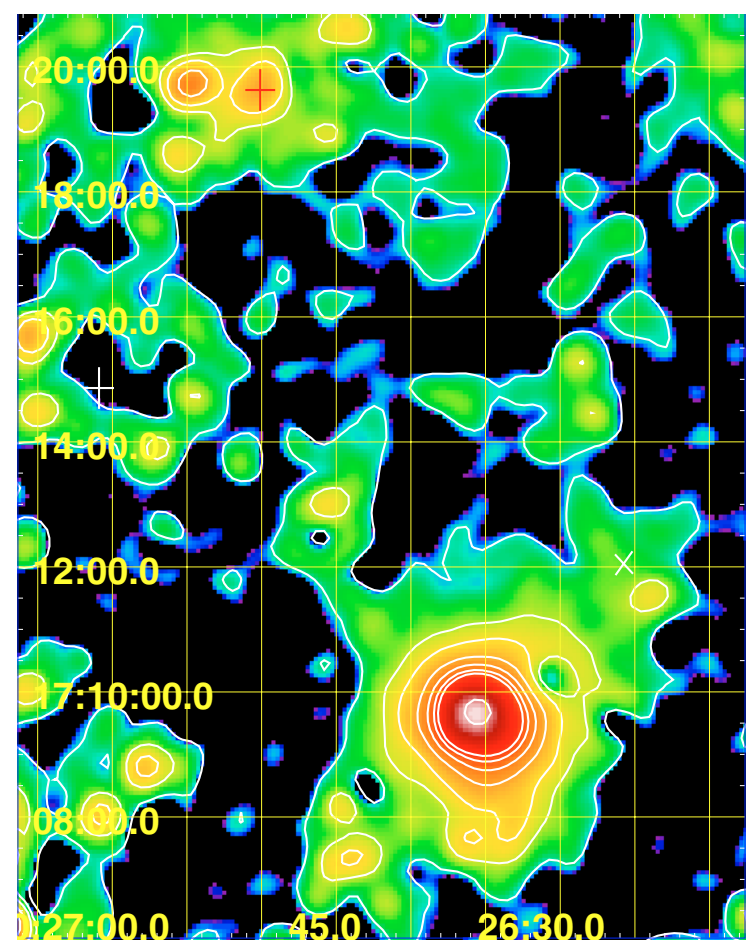

Fig. 1. Flat fielded, point sources masked and adaptively-smoothed XMM-Newton image of CL $0024+17$ in the $0.5-2 \mathrm{keV}$ band with logarithmically scaled contours. Additionally we show the galaxy pair (cross) in Czoske et al. $(2001,2002)$ and the substructure (X-point) in Kneib et al. (2003).

of $0.87 \pm 0.13 \mathrm{keV}$ fixing the metallicity to $Z=0.3 Z_{\odot}$ and a bolometric X-ray luminosity of $L_{\mathrm{X}}^{\text {bol }}=0.57 \pm 0.06 \times$ $10^{43} h_{70}^{-2} \mathrm{erg} \mathrm{s}^{-1}, 2 \%$ of the total emission of the cluster. We also observed emission from a pair of background groups in the NE which were decribed in Czoske et al. (2002) at $z=0.495$ based 

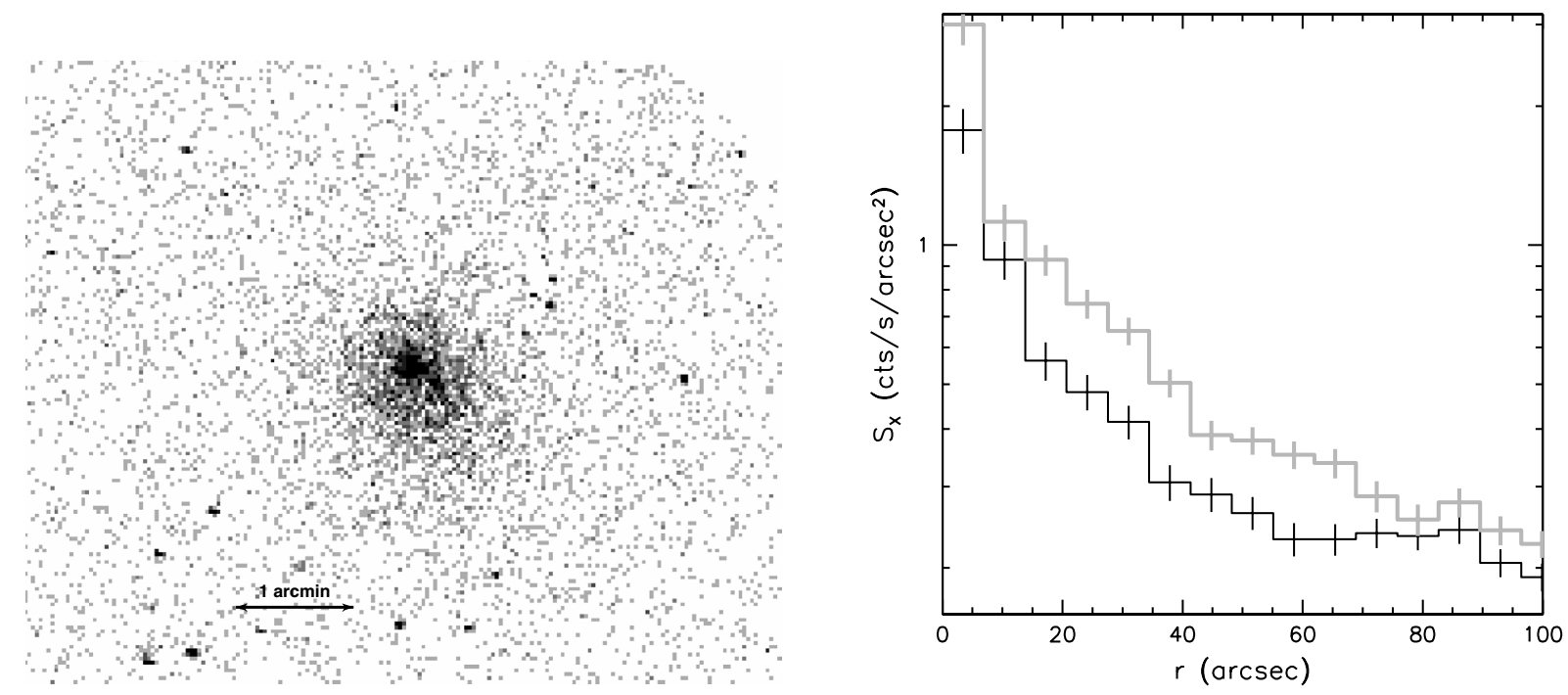

Fig. 2. Chandra image in the $0.5-2.5 \mathrm{keV}$ band (left) and surface brightness profiles (right) in annuli using the $0.5-2.5 \mathrm{keV}$ image with azimuths $30-120^{\circ}$ (counter clockwise) from north to east (black) and from south to west (grey), respectively.

on a wide-field spectroscopic survey. The northern group centered at RA $=00^{\mathrm{h}} 26^{\mathrm{m}} 50^{\mathrm{s}} .1$, Dec $=17^{\circ} 19^{\prime} 37^{\prime \prime} .8$ is more compact and X-ray luminous $\left(L_{\mathrm{X}}^{\mathrm{bol}}=0.24 \times 10^{44} h_{70}^{-2} \mathrm{erg} \mathrm{s}^{-1}\right.$, $\left.r<100^{\prime \prime}\right)$. The southern group centered at RA $=00^{\mathrm{h}} 27^{\mathrm{m}} 00^{\mathrm{s}} .9$, Dec $=17^{\circ} 14^{\prime} 51^{\prime \prime}$. 7 is too faint for spectral analysis. If we assume a gas temperature in the range $1-4 \mathrm{keV}$, then the bolometric luminosity of this group lies in the range $0.076-0.079 \times$ $10^{44} h_{70}^{-2} \operatorname{erg~s}^{-1}\left(r<100^{\prime \prime}\right)$.

\subsection{Chandra observation of the center}

CL 0024+17 was also observed by Chandra (ID: 929). Ota et al. (2004) showed an adaptively smoothed image of the cluster with a rather symmetric appearance.

A closer inspection reveals asymmetries, however. Figure 2 shows an unsmoothed image $\left(0.5-2.5 \mathrm{keV}, \sim 2^{\prime \prime}\right.$ pixels) and surface brightness profiles in annuli using the $0.5-2.5 \mathrm{keV}$ image with azimuths $30^{\circ}-120^{\circ}$ (counter clockwise) from north to east and from south to west, respectively. There is a clear asymmetry between the northeast and southwest. To the northeast, we note a sharp decrease at a radius of about $34^{\prime \prime}$. The profile to the southwest displaying a more gradual decline. In Fig. 6, we show the azimuthally averaged Chandra temperature profile from a more detailed spectral study (kindly provided by Alexey Vikhlinin) fixing the metallicity to $Z=0.3 Z_{\odot}$. We note a corresponding temperature decrease approximately at the same radius as the surface brightness decline. This complex structure including a possible indication of a "shock front" might provide further support to the cluster merger scenario.

\subsection{Optical observations}

To compare the X-ray morphology with the optical image in the central region of CL 0024+17, we retrieved 15 HST WFPC2 observations including 11 from PI: Ellis (ID: 8559) and 4 from PI: Turner (ID: 5453). They are block 0, 11, 12, 13, 18, 19, 23, 24, 25, 36, 37 and 38 in Treu et al. (2003). All images were taken in the $F 814 W$ filter ( $I$ band). We use the IRAF STSDAS and IMAGES packages to obtain the mosaic image from the calibrated science images.

The X-ray contours of the XMM-Newton observations were plotted on the merged HST image (shown in Fig. 3). We note the overlapping cluster centers and galaxy concentrations in the NW $\left(\sim 3.3^{\prime}\right)$ of the X-ray and optical images.

\subsection{Hardness ratio maps}

To further study the substructure in CL $0024+17$ we produced hardness ratio map (HRM) of the cluster, which primarily reflects the temperature distribution. The hardness ratio is the photon flux ratio of the hard band of $2-7.5 \mathrm{keV}$ to the soft band of $0.5-2 \mathrm{keV}, H R=I(2-7.5 \mathrm{keV}) / I(0.5-2 \mathrm{keV})$. An indication of an elongation in the NW-SE direction is visible in the HRM (see Fig. 4) on a scale of $r \sim 3.3^{\prime}$. The HRM shows a complicated structure with an average $H R$ of $0.55 \pm 0.39$ in the central $r<3^{\prime}$ region. The substructure in the NW $\left(\sim 3.3^{\prime}\right)$ has an average of $H R=0.48 \pm 0.34$ within $1^{\prime}$. We note two peaks: one is at $\left(\mathrm{RA}=00^{\mathrm{h}} 26^{\mathrm{m}} 24^{\mathrm{s}} .8\right.$, Dec $\left.=17^{\circ} 11^{\prime} 13^{\prime \prime} .2\right)$ that is marginally coincident with the NW substructure; the other is close to the center at RA $=00^{\mathrm{h}} 26^{\mathrm{m}} 30^{\mathrm{s}} \cdot 9$, Dec $=17^{\circ} 09^{\prime} 50^{\prime} \cdot 3$.

\subsection{Temperature and metallicity profiles}

We first derive global X-ray properties of CL 0024+17 from the spectra extracted from the $r<3^{\prime}$ region, covering radii up to a spherical overdensity of $\sim 250$, i.e. the ratio of the mean density of the dark halo with respect to the redshift-dependent critical density $\rho_{\text {crit }}(z)$. We measure an emission weighted temperature of $3.52 \pm 0.17 \mathrm{keV}$, metallicity of $0.22 \pm 0.07$ and bolometric luminosity of $2.9 \pm 0.1 \times 10^{44} h_{70}^{-2} \mathrm{erg} \mathrm{s}^{-1}\left(\chi^{2} /\right.$ d.o.f. $=$ $248.5 / 228)$. We confirm the estimates of $3-4.5 \mathrm{keV}$ and $L_{\mathrm{X}}^{\text {bol }}=$ $2.40 \times 10^{44} h_{70}^{-2} \mathrm{erg} \mathrm{s}^{-1}$ in Böhringer et al. (2000) from the ROSAT observation and a estimate of $5.7_{-2.1}^{+4.2} \mathrm{keV}$ in Soucail et al. (2000) from the ASCA observations. The Chandra 


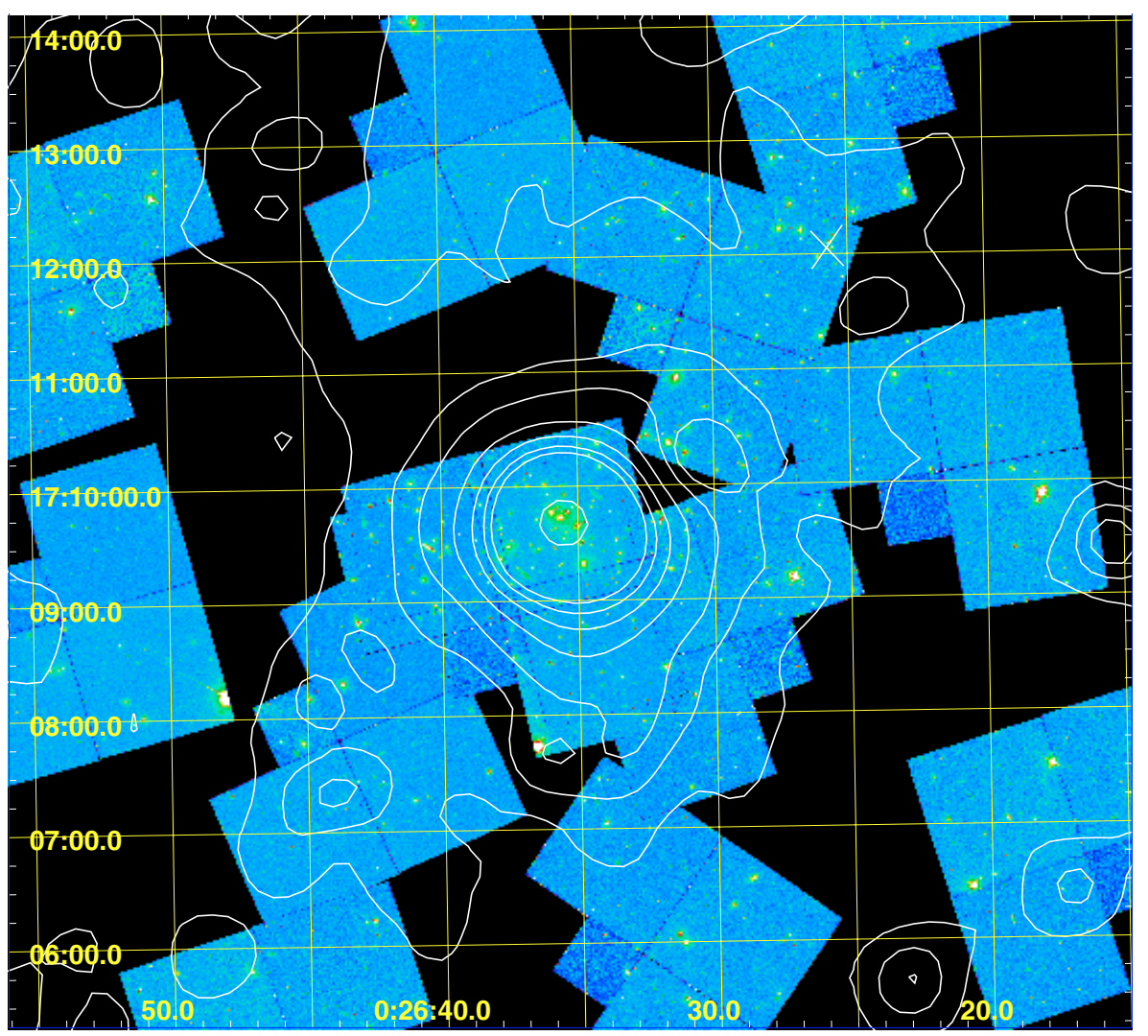

Fig. 3. HST mosaic image of the X-ray center of CL 0024+17. Superposed X-ray contours of XMM-Newton as shown in Fig. 1 indicate the elongation in the NW-SE direction on the scale of $r \sim 3.3^{\prime}$ (X-point).

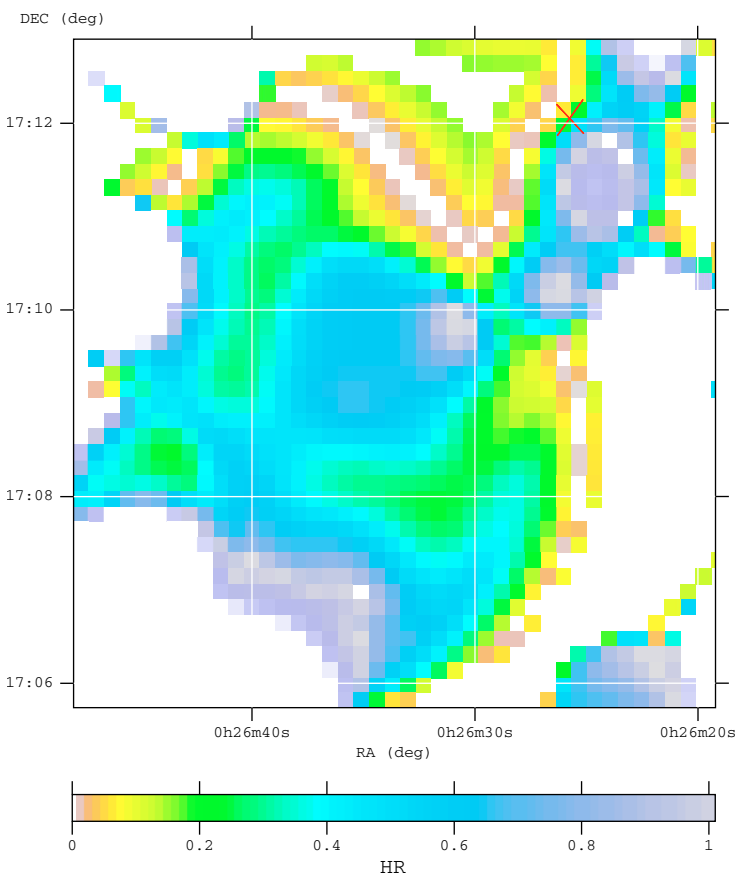

Fig. 4. XMM-Newton HRM ( 10" pixels) of CL 0024+17. Additionally we show the position of the substructure (X-point) in Kneib et al. (2003).

observations yield a comparable temperature of $4.47_{-0.27}^{+0.42} \mathrm{keV}$ and a bolometric luminosity of $2.60 \times 10^{44} h_{70}^{-2} \mathrm{erg} \mathrm{s}^{-1}$

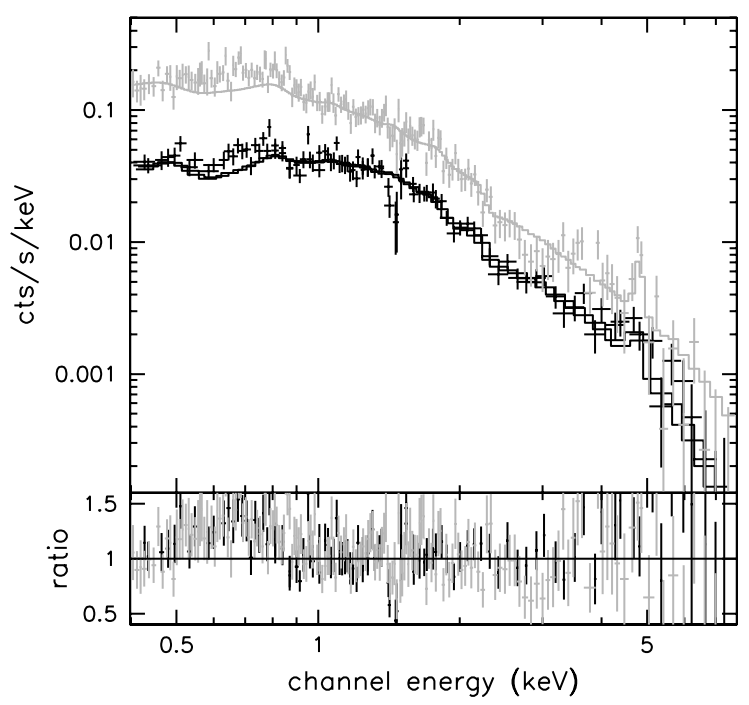

Fig. 5. XMM-Newton spectra (top panel) extracted from the $<3^{\prime}$ region fitted in the $1-10 \mathrm{keV}$ band by an isothermal model and their residuals (bottom panel) for MOS (black) and pn (grey), respectively.

(Ota et al. 2004). The source spectra for $r<3^{\prime}$ and the fitted model are shown in Fig. 5. A lower luminosity of $1.26 \times$ $10^{44} h_{70}^{-2} \mathrm{erg} \mathrm{s}^{-1}$ is predicted from the $L-T$ relation in Arnaud \& Evrard (1999) and the XMM-Newton temperature measurement. The high luminosity might indicate the complex cluster center which will be discussed in Sect. 4 . 

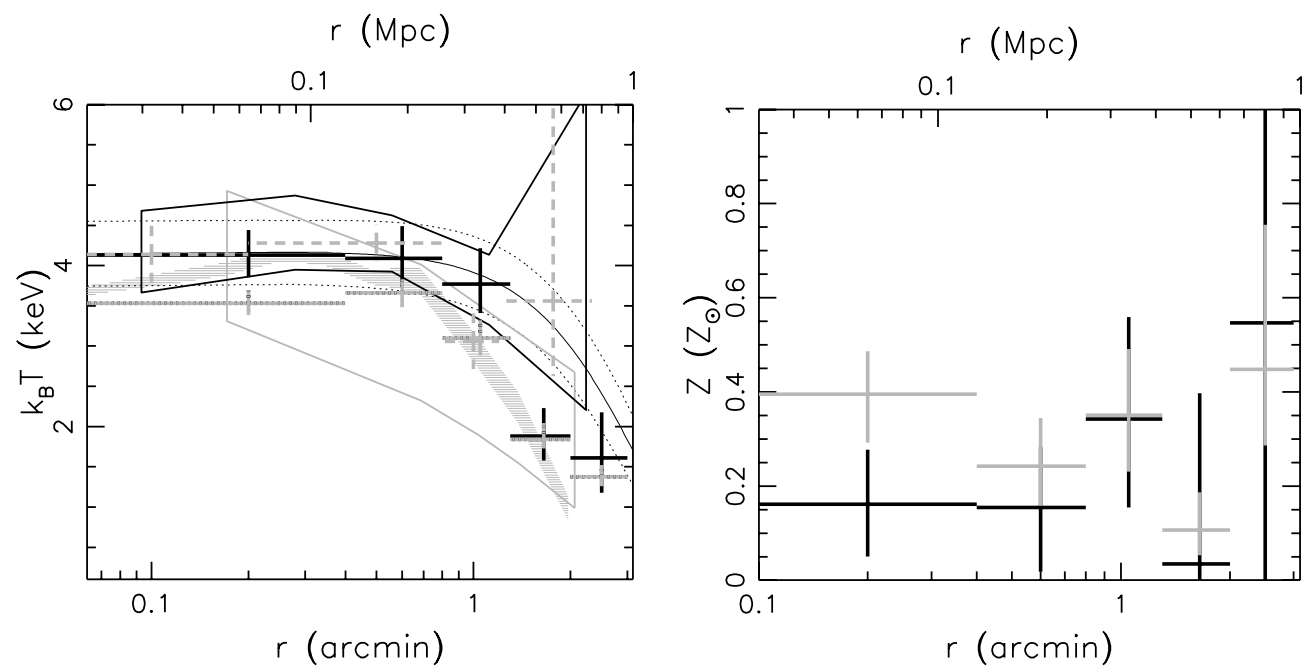

Fig. 6. Projected temperature (left) and metallicity (right) profiles measured from the XMM-Newton data in the 1-7.8 keV band (solid, black) and $0.4-7.8 \mathrm{keV}$ band (solid, grey) with point sources excluded. A solid curve in black indicates the best Gaussian fit of the temperature measurements in the 1-7.8 keV band with confidence intervals as dotted curves. Additionally the similarity temperature profiles in Markevitch et al. (1998; grey) and in Zhang et al. (2004b; black) are indicated by solid outlines. Our Chandra temperature measurements of CL 0024+17 (dashed, grey) and the temperature profile of Sersic 159-03 (grey shadow; $1 \sigma$ interval) in Kaastra et al. (2001) scaled to CL 0024+17 are presented for comparison.

For a more detailed spectral study we divide the cluster region into five annuli: $0-0.4^{\prime}, 0.4-0.8^{\prime}, 0.8-1.3^{\prime}, 1.3-2^{\prime}$, and $2-3^{\prime}$. We investigate the dependence of the temperature and metallicity measurements on the low energy (low-E) cut-off $(0.4 \mathrm{keV}$ or $1 \mathrm{keV})$ as we did in our earlier study (Zhang et al. 2004a) to test the robustness of the spectral data fitting. We find that in the $r<1^{\prime}$ region the derived temperatures increase by $15 \%$ if the low-E cut-off is increased from $0.4 \mathrm{keV}$ to $1 \mathrm{keV}$. With the decreasing signal-noise-ratio $(S / N)$ in the $r>1^{\prime}$ region the derived temperatures increase by up to $40 \%$ if the lowE cut-off is increased from $0.4 \mathrm{keV}$ to $1 \mathrm{keV}$. As in our earlier work (see also Pratt \& Arnaud 2002) we attribute this change in the best fit temperature with energy range to the influence of a contaminating soft spectral component. To minimize its effect, we use the higher energy cut-off at $1 \mathrm{keV}$ above which the results are stable.

The radial metallicity distribution is very flat which is expected from simulations where merging efficiently flattens the metallicity profile (Kobayashi 2004). The deprojection correction does not provide a significant change in the results. In the $r<1^{\prime}\left(r>1^{\prime}\right)$ region the temperature measurements vary by a factor of 5\% (50\%), which is approximately within the error bars. The temperature and metallicity profiles determined from the spectra in the 5 annuli $\left(\chi^{2} /\right.$ d.o.f. $=$ $68.2 / 72,77.0 / 78,64.0 / 60,50.2 / 60,110.1 / 96$ from the inner to the outer annulus) are shown in Fig. 6. A variation of the temperature with radius is found here for the first time for CL 0024+17. The inner three bins covering the radial range to a radius of $1.3^{\prime}\left(416 h_{70}^{-1} \mathrm{kpc}\right)$ are consistent with an isothermal profile. This agrees with the Chandra results by Ota et al. (2004) who found an isothermal temperature profile to an outer radius of $1.5^{\prime}\left(480 h_{70}^{-1} \mathrm{kpc}\right)$. The form of the temperature profile is very similar to the profile we obtained for the sample of REFLEX-DXL (ROSAT-ESO Flux-Limited X-ray cluster survey, Distant X-ray Luminous) clusters (Zhang et al. 2004b). We scaled the temperature profile in Markevitch et al. (1998) to CL 0024+17 using a radius of $r_{180}=1.10 h_{70}^{-1} \mathrm{Mpc}$ (obtained in Sect. 3.9) and an emission weighted global temperature of $3.52 \mathrm{keV}$. We find good overall agreement as found for the REFLEX-DXL clusters except that the flat part of the temperature profile extends to a larger radius than the average profile of Markevitch et al. (1998). We also scaled the temperature profile for Sersic 159-03 in Kaastra et al. (2001) to CL 0024+17, and found a similarly rapid temperature drop as a function of radius as CL 0024+17.

We found an isothermal temperature of $\sim 3.9 \mathrm{keV}$ to a radius of $1.5^{\prime}\left(480 h_{70}^{-1} \mathrm{kpc}\right)$ and a power law model with an index $\gamma=0.98$ outside this radius. We approximate the temperature profile with two analytical models: (a) an isothermal model using the global temperature of $3.52 \pm 0.17 \mathrm{keV}$; (b) a function with the shape of a Gaussian of $k_{\mathrm{B}} T(r)=k_{\mathrm{B}} T_{\mathrm{g}} \cdot \mathrm{e}^{-\frac{1}{2}\left(\frac{r-r_{\mathrm{g}}}{w}\right)^{2}}$ (see Table 5) which approximately fits the observed temperature profile.

\subsection{Surface brightness}

We obtained an azimuthally averaged surface brightness profile for CL $0024+17$ in the $0.5-2 \mathrm{keV}$ band for each instrument, in which the central position is RA $=00^{\mathrm{h}} 26^{\mathrm{m}} 35^{\mathrm{s}} .7$, Dec $=17^{\circ} 09^{\prime} 35^{\prime \prime} 8$. This energy band is selected because it provides an almost temperature-independent X-ray emission coefficient over the expected temperature range. After the vignetting correction and double-step background subtraction described above, we find that a $\beta$-model (e.g. Cavaliere \& Fusco-Femiano 1976; Jones \& Forman 1984)

$S_{x}(r)=S_{0}\left(1+\frac{r^{2}}{r_{\mathrm{c}}^{2}}\right)^{-3 \beta+1 / 2}$ 


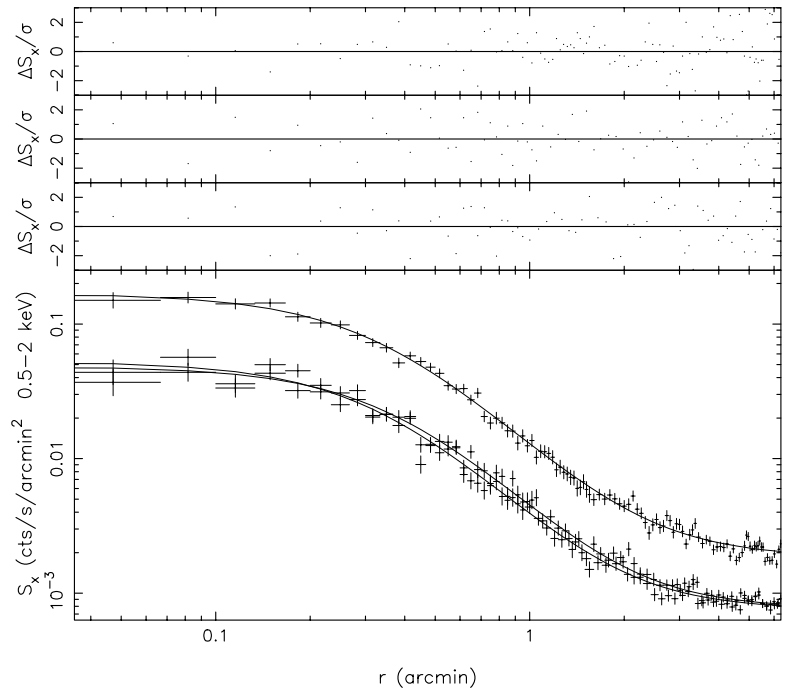

Fig. 7. Flat fielded, point source subtracted and azimuthally averaged radial surface brightness profiles for CL $0024+17$ in the $0.5-2 \mathrm{keV}$ band for pn (top) and MOS (bottom) and their best $\chi^{2}$ fits by a PSF convolved $\beta$-model combining a constant residual soft X-ray background. Residuals scaled by the data uncertainties appear in the upper three panels for MOS1, MOS2 and pn, respectively, from top to bottom.

convolved with the XMM-Newton PSF provides an adequate $\chi^{2}$ fit to the surface brightness profile (Fig. 7). A PSF at each position for each instrument is determined from an empirical calibration (Ghizzardi 2001) according to a mean energy $\sim 1.25 \mathrm{keV}$ of the $0.5-2 \mathrm{keV}$ band and its off-axis radius.

We compare the PSF convolved and unconvolved $\beta$-model parameters in Table 3, and find that the core radii are overestimated and the slope parameters are underestimated in the latter case. The combined $90 \%$ confidence contours (see Fig. 8) of the three instruments provide a narrow range for $r_{\mathrm{c}}$ and $\beta$ $\left(0.266<r_{\mathrm{c}}<0.295^{\prime} ; 0.551<\beta<0.593\right)$ at the $90 \%$ confidence level. Böhringer et al. (2000) obtained $r_{\mathrm{c}}=0.11-0.28^{\prime}$ and $\beta=0.425-0.550$ from the ROSAT HRI data corrected for PSF. Ota et al. (2004) obtained $r_{\mathrm{c}}=0.287 \pm 0.014^{\prime}$ and $\beta=0.55 \pm 0.02$ from the Chandra observations. Our result confirms the results from previous X-ray observations with a higher accuracy because of the improvement of the image quality of XMM-Newton compared to previous observations.

The surface brightness profile approximated by a $\beta$-model can be analytically deprojected to yield the emission per volume element, $\xi(r)=\widetilde{\Lambda}(r) n_{\mathrm{e}}^{2}(r)$. With the given emissivity, $\widetilde{\Lambda}(r)$, from the applied plasma model, one can derive the electron density profile $n_{\mathrm{e}}(r)=n_{\mathrm{e} 0}\left(1+\frac{r^{2}}{r_{\mathrm{c}}^{2}}\right)^{-3 \beta / 2}$ with the parameters given in Table 3 combining the three detectors. We studied the influence of the central emission in the beta model analysis and found that the parameters like $\beta$ and $r_{\mathrm{c}}$ change within $2 \%$ if the central bins are masked.

We obtain the pressure distribution $P(r)$ as a function of radius directly from the temperature and electron density measurements. Thus a $\beta$-model is applied to fit the pressure with results shown in Table 5 .

Furthermore, we simulated a symmetric, background subtracted and flat fielded image using the parameters of

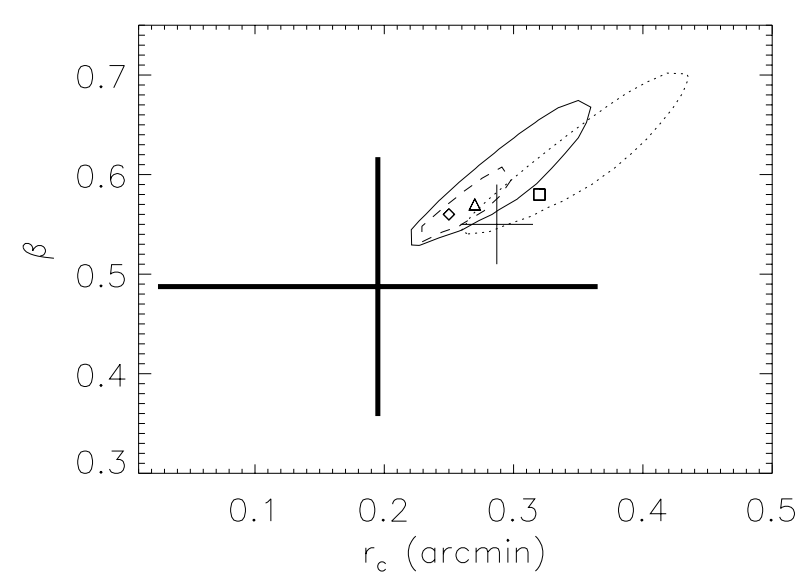

Fig. 8. 90\% confidence contours and the best fit values for the core radius $r_{\mathrm{c}}$ and slope parameter $\beta$ of the $\beta$ model for the surface brightness of XMM-Newton for MOS1 (solid; triangle), MOS2 (dotted; square) and pn (dashed; diamond), respectively. Chandra (thin; Ota et al. 2004) and ROSAT (thick; Böhringer et al. 2000) measurements are also shown as crosses.

the $\beta$-model in Table 3 . The residual map, which contains the background, is obtained by extracting the simulated image from the adaptively-smoothed image. We confirm the existence of excess emission in the substructure in the NW, while the negative residual surface brightness in the cluster center shows that the elongation flattens the surface brightness compared to a symmetric structure.

To further test if the cluster mass profile can be described by a NFW profile (Navarro et al. 1997; NFW), we fit the observed surface brightness profile by the model computed from the NFW model described dark matter halo (e.g. Makino et al. 1998). A model that fits the data well over a large range of radii shows an inner cusp in the surface brightness. The central surface brightness model at the given resolution is about a factor of two to three higher and thus inconsistent with our observations. Since we detect signatures of a cluster merger in the central region. We are not surprised to find deviations from the NFW model. Also Tyson et al. (1998) found a core in the mass profile in their lensing analysis.

\subsection{Cooling time of the gas}

The cooling time is the total energy of the gas divided by the energy loss rate (e.g. Zhang \& Wu 2003)

$t_{\mathrm{c}}=2.869 \times 10^{10} \mathrm{yr}\left(\frac{1.2}{g}\right)\left(\frac{k_{\mathrm{B}} T}{\mathrm{keV}}\right)^{\frac{1}{2}}\left(\frac{n_{\mathrm{e}}}{10^{-3} \mathrm{~cm}^{-3}}\right)^{-1}$,

where $n_{\mathrm{e}}$ and $k_{\mathrm{B}} T$ are the electron number density and temperature, respectively, and $g$ is the Gaunt factor (a function of $k_{\mathrm{B}} T$ ). The resulting cooling time as a function of radius is shown in Fig. 9. The central cooling time of $4.5 \mathrm{Gyr}$ is smaller than the age of the Universe and probably smaller than the age of the cluster if we assume that the age of the cluster mass concentraion is longer than two crossing time because the merger frequency is expected to be a few Gyr (e.g. Schuecker et al. 2001). An even smaller central cooling time is implied by the Chandra data (Ota et al. 2004) due to the higher angular resolution of 
Table 3. Parameters of the $\beta$-model. Column (1): instrument; Col. (2): "Y" or "N" means the PSF convolved or unconvolved $\beta$-model; Col. (3): central surface brightness (0.5-2 keV) in cts s ${ }^{-1} \operatorname{arcmin}^{-2}$; Col. (4): core radius in arcmin; Col. (5): slope parameter; Col. (6): $\chi^{2} /$ d.o.f.

\begin{tabular}{lccccl}
\hline \hline & PSF & $S_{0}$ & $r_{\mathrm{c}}$ & $\beta$ & $\chi^{2} /$ d.o.f. \\
\hline MOS1 & $\mathrm{Y}$ & $0.106 \pm 0.015$ & $0.27 \pm 0.03$ & $0.57 \pm 0.02$ & $99.7 / 63$ \\
& $\mathrm{~N}$ & $0.047 \pm 0.004$ & $0.34 \pm 0.03$ & $0.56 \pm 0.01$ & $204.3 / 150$ \\
MOS2 & $\mathrm{Y}$ & $0.094 \pm 0.010$ & $0.32 \pm 0.03$ & $0.58 \pm 0.02$ & $79.2 / 65$ \\
& $\mathrm{~N}$ & $0.045 \pm 0.003$ & $0.36 \pm 0.03$ & $0.54 \pm 0.01$ & $159.7 / 150$ \\
pn & $\mathrm{Y}$ & $0.375 \pm 0.033$ & $0.25 \pm 0.02$ & $0.56 \pm 0.01$ & $130.5 / 88$ \\
& $\mathrm{~N}$ & $0.158 \pm 0.007$ & $0.33 \pm 0.02$ & $0.54 \pm 0.01$ & $206.3 / 150$ \\
\hline
\end{tabular}

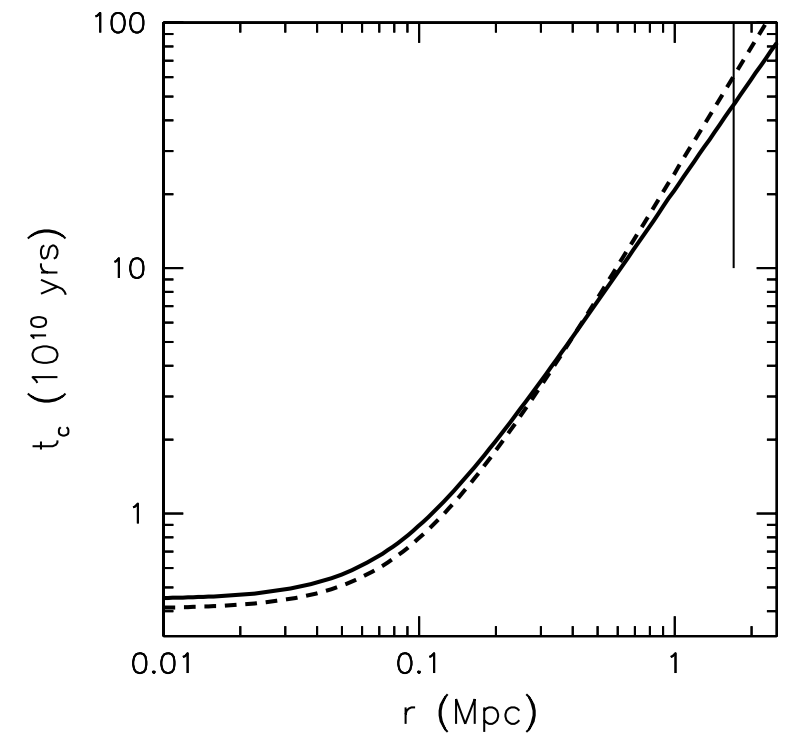

Fig. 9. Cooling time of the gas using the global temperature (dashed) and using the observed temperature profile (solid). The vertical line indicates $r_{200}=1.7 h_{70}^{-1} \mathrm{Mpc}$, predicted from the lensing data in Kneib et al. (2003).

the central region. The data thus correspond, in the classical interpretation, to a small or moderate cooling flow (Fabian \& Nulsen 1977).

\subsection{Gas entropy}

The main part of the observed entropy, defined as $S=k_{\mathrm{B}} T n_{\mathrm{e}}^{-2 / 3}$ (e.g. Ponman et al. 1999), results from shock heating of the gas during cluster formation and it scales with the cluster critical temperature. An excess above this scaling law indicates the effect of an additional, non-gravitational heating source (e.g. Lloyd-Davies et al. 2000). While a low central entropy indicates significant radiative cooling. We show the entropy profile derived for CL 0024+17 from the XMM-Newton observations in Fig. 10.

The entropy of the $r>0.1 h_{70}^{-1} \mathrm{Mpc}$ region derived from temperature measurements of CL $0024+17$ has a similar slope as that predicted from a spherical accretion shock model, $S \propto$ $r^{1.1}$ (Kay 2004; thick grey line in Fig. 10). The entropy under the assumption of isothermality becomes steeper than the entropy derived from temperature measurements, especially in the outer region.
The entropy of the gas in the center $\left(r<0.1 h_{70}^{-1} \mathrm{Mpc}\right)$ lies below the entropy floor, $S \sim 124 h_{70}^{-1 / 3} \mathrm{keV} \mathrm{cm}^{2}$, derived for clusters by Lloyd-Davies et al. (2000). This indicates some effect from radiative cooling.

We scale the entropy of CL $0024+17$ by $1+\frac{k_{\mathrm{B}} T(r)}{k_{\mathrm{B}} T_{0}}$, where $k_{\mathrm{B}} T_{0}=2 \mathrm{keV}$ is a constant related to the degree of preheating. In Fig. 10, we compare it to the entropy of the Birmingham-CfA clusters in the temperature range of 2.9$4.6 \mathrm{keV}$ (Ponman et al. 2003) scaled also by $1+\frac{k_{\mathrm{B}} T(r)}{k_{\mathrm{B}} T_{0}}$. The entropy of CL 0024+17 agrees with the self-similarly scaled entropy described by Ponman et al. (2003) within the observed dispersion of their cluster sample.

Equation (2) can be rewritten as

$S_{\mathrm{c}}=100 \mathrm{keV} \mathrm{cm}^{2}\left(\frac{t_{\mathrm{c}}}{2.869 \times 10^{10} \mathrm{yr}}\right)^{\frac{2}{3}}\left(\frac{g}{1.2}\right)^{\frac{2}{3}}\left(\frac{k_{\mathrm{B}} T}{\mathrm{keV}}\right)^{\frac{2}{3}}$.

If the cooling time $t_{\mathrm{c}}$ is chosen approximately to be the age of the Universe, the above equation would allow us to determine the critical entropy floor $S_{\mathrm{c}}$ of the gas in the cluster before the onset of significant cooling and possible mass deposition. In the center of CL $0024+17$ the observed entropy is slightly below this value $\left(\sim 154 \mathrm{keV} \mathrm{cm}{ }^{2}\right)$ which implies a insignificant radiative cooling.

\subsection{Mass modeling}

We assume the intracluster gas to be in hydrostatic equilibrium within the gravitational potential dominated by dark matter. Neglecting the cosmological constant, $\Lambda$, at these high overdensities (a less than $1 \%$ effect, see Zhang et al. 2004a), we have

$\frac{1}{\mu m_{\mathrm{p}} n_{\mathrm{e}}} \frac{\mathrm{d}\left(n_{\mathrm{e}} k_{\mathrm{B}} T\right)}{\mathrm{d} r}=-\frac{G M(r)}{r^{2}}$,

where $\mu=0.62$ is the mean molecular weight per hydrogen atom.

Analytic models of the gas density and pressure profiles derived above can be easily combined with Eq. (4) to obtain the mass profile (Fig. 11). The virial radius (not $r_{200}$ ) is defined to be a radius with overdensity (the average density with respect to $\rho_{\text {crit }}$ at the cosmic epoch $z$ ) of $\Delta_{\mathrm{c}}=18 \pi^{2}+$ $82\left[\Omega_{\mathrm{m}}(z)-1\right]-39\left[\Omega_{\mathrm{m}}(z)-1\right]^{2}$ for a flat universe. $\Omega_{\mathrm{m}}(z)$ is the cosmic density parameter (e.g. Zhang \& Wu 2003). Therefore the mass profiles in Fig. 11 combined with the expression for the overdensity yield $r_{\text {vir }}=1.37 h_{70}^{-1} \mathrm{Mpc}$ using the global 

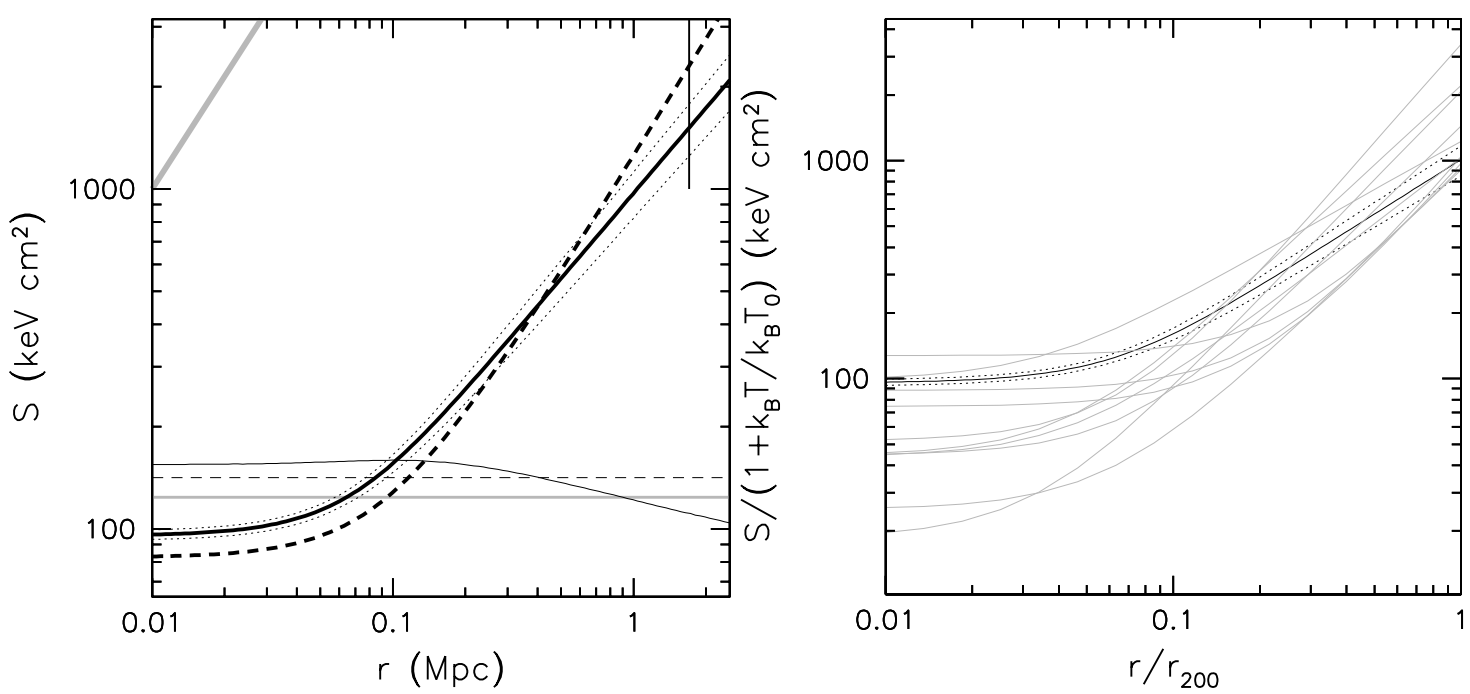

Fig. 10. Left: entropy (thick, black) and critical entropy floor (thin, black) using the global temperature (dashed) and using the observed temperature profile (solid, confidence intervals as dotted curves). Additional grey lines are the entropy floor (thin), $S \sim 124 h_{70}^{-1 / 3} \mathrm{keV} \mathrm{cm}^{2}$, from Lloyd-Davies et al. (2000) and the predicted slope of 1.1 (thick) from the spherical accretion shock model (Kay 2004). The vertical line indicates the radius as defined in Fig. 9. Right: scaled entropy of CL $0024+17$ vs. scaled radius using the radius of $r_{200}=1.05 h_{70}^{-1} \mathrm{Mpc}$ (black, confidence intervals as dotted curves) and the Birmingham-CfA clusters in a temperature range of $2.9-4.6 \mathrm{keV}$ (grey).

Table 4. Comparison of the gravitational mass from the X-ray and optical lensing measurements.

\begin{tabular}{|c|c|c|c|c|c|c|}
\hline \multicolumn{2}{|c|}{ Observation } & \multicolumn{2}{|c|}{$\overline{\text { XMM-Newton }}^{a}$} & \multirow{2}{*}{$\begin{array}{l}\text { Chandra }^{b} \\
1.4\end{array}$} & \multicolumn{2}{|c|}{ "HST } \\
\hline$r_{200}$ & $\left(h_{70}^{-1} \mathrm{Mpc}\right)$ & 1.11 & 1.05 & & 1.7 & $c$ \\
\hline$M_{200}$ & $\left(10^{14} h_{70}^{-1} M_{\odot}\right)$ & $2.3 \pm 0.1$ & $2.0 \pm 0.3$ & $4.6_{-0.5}^{+0.7}$ & $5.7_{-1.0}^{+1.1}$ & c \\
\hline$M_{\mathrm{proj}}(r=0.143)$ & $\left(10^{14} h_{70}^{-1} M_{\odot}\right)$ & $0.61 \pm 0.01$ & $0.48 \pm 0.03$ & - & $1.59 \pm 0.04$ & $d$ \\
\hline$M_{\text {proj }}(r=0.153)$ & $\left(10^{14} h_{70}^{-1} M_{\odot}\right)$ & $0.64 \pm 0.01$ & $0.51 \pm 0.03$ & $\begin{array}{l}0.60_{-0.09}^{+0.14}(\beta) \\
0.53_{-0.08}^{+0.13} \text { (NFW) }\end{array}$ & $2.37 \pm 0.36$ & $e$ \\
\hline$M(r=1.4)$ & $\left(10^{14} h_{70}^{-1} M_{\odot}\right)$ & $3.0 \pm 0.2$ & $2.4 \pm 0.3$ & $4.6_{-0.5}^{+0.00}$ & - & \\
\hline$M(r=1.7)$ & $\left(10^{14} h_{70}^{-1} M_{\odot}\right)$ & $3.6 \pm 0.2$ & $2.8 \pm 0.4$ & - & $5.7_{-1.0}^{+1.1}$ & $c$ \\
\hline
\end{tabular}

${ }^{a}$ XMM-Newton results using the global temperature and using the observed temperature profile in which projected masses use the truncation radius of $r_{200}=2.5 h_{70}^{-1} \mathrm{Mpc} ;{ }^{b}$ Ota et al. (2004); ${ }^{c}$ Kneib et al. (2003); ${ }^{d}$ Broadhurst et al. (2000); ${ }^{e}$ Tyson et al. (1998).

temperature and $r_{\mathrm{vir}}=1.26 h_{70}^{-1} \mathrm{Mpc}$ using the observed temperature distribution.

We compare the XMM-Newton results with the recent measurements from Chandra and HST in Table 4 and Fig. 11. The XMM-Newton measurements using the measured temperature profile are slightly lower than Chandra values derived under the assumption of isothermality. As shown in Table 4 and Fig. 11, the discrepancy between the X-ray measured total mass and strong lensing mass at the radii of $0.143 h_{70}^{-1} \mathrm{Mpc}$ (Broadhurst et al. 2000) and $0.153 h_{70}^{-1} \mathrm{Mpc}$ (Tyson et al. 1998) remains large, about a factor of 4 .

Under the assumption of isothermality, the XMM-Newton measurements are more consistent with the observationally determined $M_{200}-T$ relation,

$M_{200}=4.11 \pm 0.02 \times 10^{13} h_{70}^{-1}\left(\frac{k T_{\mathrm{B}}}{\mathrm{keV}}\right)^{1.60 \pm 0.04} M_{\odot}$,

which is based on the conventional $\beta$ model for the X-ray surface brightness profile and hydrostatic equilibrium for 22 nearby clusters from Xu et al. (2001). For CL 0024+17, Eq. (5) provides $M_{200}=3.08 \times 10^{14} h_{70}^{-1} M_{\odot}$ using the X-ray temperature. The masses obtained from the X-ray temperature measurement via the $M-T$ relations in Finoguenov et al. (2001) and in Bryan \& Norman (1998) are $M_{200}=5.96 \times 10^{14} h_{70}^{-1} M_{\odot}$ and $M_{200}=3.76 \times 10^{14} h_{70}^{-1} M_{\odot}$ in which the former is more consistent with the HST optical lensing estimate.

Navarro et al. (1997; NFW) describe a universal density profile from numerical simulations in hierarchical clustering scenarios

$\rho_{\mathrm{DM}}(r)=\delta_{\text {crit }} \rho_{\text {crit }}\left(\frac{r}{r_{\mathrm{s}}}\right)^{-1}\left(1+\frac{r}{r_{\mathrm{s}}}\right)^{-2}$,

where $\delta_{\text {crit }}$ and $r_{\mathrm{s}}$ are the characteristic density and scale of the halo, respectively, $\rho_{\text {crit }}$ is the critical density of the Universe at the cosmic epoch $z$ and $\rho_{\mathrm{s}}=\delta_{\text {crit }} \rho_{\text {crit }} . \delta_{\text {crit }}$ is related to the concentration parameter of a dark halo $c=r_{\mathrm{vir}} / r_{\mathrm{s}}$ by

$\delta_{\text {crit }}=\frac{200}{3} c^{3}\left[\ln (1+c)-\frac{c}{1+c}\right]^{-1}$.

We fit the X-ray determined mass profile with a NFW model, and found that the NFW model does not provide a good fit to our data in the $r<0.1 h_{70}^{-1} \mathrm{Mpc}$ region (Fig. 11). However the 

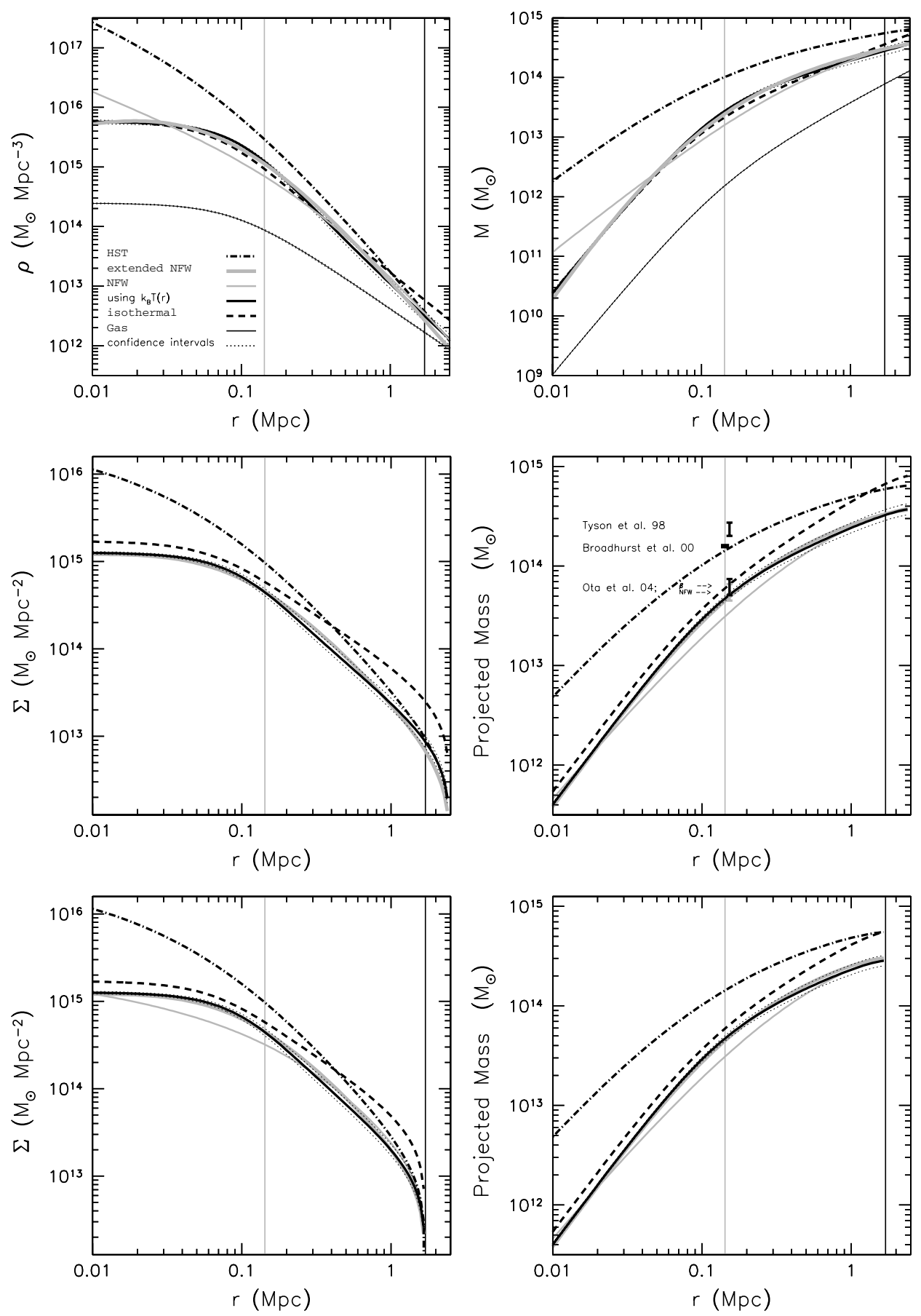

Fig. 11. Upper left: dark matter density and gas density, upper right: gravitational mass and gas mass, middle left: projected dark matter density $\left(r_{\text {truncate }}=2.5 h_{70}^{-1} \mathrm{Mpc}\right)$, middle right: projected gravitational mass $\left(r_{\text {truncate }}=2.5 h_{70}^{-1} \mathrm{Mpc}\right)$, lower left: projected dark matter density $\left(r_{\text {truncate }}=1.7 h_{70}^{-1} \mathrm{Mpc}\right)$, lower right: projected gravitational mass $\left(r_{\text {truncate }}=1.7 h_{70}^{-1} \mathrm{Mpc}\right)$. The meaning of the lines is: (i) hydrostatic equilibrium (black) using the global temperature (thick, dashed) and using the observed temperature profile (thick, solid, confidence intervals as dotted curves) and its best fit (grey) of extended NFW model (thick) and NFW model (thin); (ii) HST lensing measurements (Kneib et al. 2003; dash-dotted). Additionally we present the gas density and gas mass (thin, black) with confidence intervals as dotted curves. We label the strong lensing projected gravitational mass of $1.59 \pm 0.04 \times 10^{14} h_{70}^{-1} M_{\odot}$ (Broadhurst et al. 2000) and $2.37 \pm 0.36 \times 10^{14} h_{70}^{-1} M_{\odot}$ (Tyson et al. 1998) at the arc radii of $0.143 h_{70}^{-1} \mathrm{Mpc}$ and $0.153 h_{70}^{-1} \mathrm{Mpc}$ and the Chandra results (Ota et al. 2004) using a $\beta / \mathrm{NFW}$ model at the arc radius of $0.153 h_{70}^{-1} \mathrm{Mpc}$ for comparison. The black vertical line indicates the radius as defined in Fig. 9. The grey vertical line indicates the arc radius of $0.143 h_{70}^{-1} \mathrm{Mpc}$ (e.g. Broadhurst et al. 2000).

NFW model fit provides a virial radius of $c r_{\mathrm{s}}=1.28 h_{70}^{-1} \mathrm{Mpc}$ which is consistent with the virial radii of $r_{\mathrm{vir}}=1.37 h_{70}^{-1} \mathrm{Mpc}$ and $r_{\mathrm{vir}}=1.26 h_{70}^{-1} \mathrm{Mpc}$ derived from the mass profiles using the global temperature and using the observed temperature distribution, respectively, in Fig. 11 combined with the expression for the overdensity. We derive a radius of $r_{200}=0.93 h_{70}^{-1} \mathrm{Mpc}$ 
Table 5. Parameters of each profile model for the best $\chi^{2}$ fit.

\begin{tabular}{lll}
\hline \hline Model & \multicolumn{2}{c}{ Parameter } \\
\hline Gaussian & $k_{\mathrm{B}} T_{g}(\mathrm{keV})$ & $4.2 \pm 0.4$ \\
& $r_{g}\left(h_{70}^{-1} \mathrm{Mpc}\right)$ & $0.08 \pm 0.04$ \\
& $w$ & $0.69 \pm 0.04$ \\
$\beta$ & $r_{\mathrm{c}}^{n_{\mathrm{e}}}\left(h_{70}^{-1} \mathrm{Mpc}\right)$ & $0.093 \pm 0.001$ \\
& $n_{\mathrm{e} 0}\left(10^{-3} \mathrm{~cm}^{-3}\right)$ & $8.8 \pm 0.1$ \\
& $\beta^{n_{\mathrm{e}}}$ & $0.57 \pm 0.01$ \\
& $r_{\mathrm{c}}^{\mathrm{P}}\left(h_{70}^{-1} \mathrm{Mpc}\right)$ & $0.11 \pm 0.01$ \\
& $P_{0}\left(10^{-2} \mathrm{keV} \mathrm{cm}^{-3}\right)$ & $3.59 \pm 0.04$ \\
& $\beta^{\mathrm{P}}$ & $0.68 \pm 0.01$ \\
& $r_{\mathrm{s}}\left(h_{70}^{-1} \mathrm{Mpc}\right)$ & $0.37 \pm 0.03$ \\
& $\rho_{\mathrm{s}}\left(10^{14} M_{\odot} \mathrm{Mpc}^{-3}\right)$ & $5.3 \pm 0.7$ \\
& $\mathrm{c}$ & 3.5 \\
Extended NFW & $r_{\mathrm{s}}\left(h_{70}^{-1} \mathrm{Mpc}\right)$ & $0.072 \pm 0.004$ \\
& $\rho_{\mathrm{s}}\left(10^{16} M_{\odot} \mathrm{Mpc}^{-3}\right)$ & $4.3 \pm 1.7$ \\
& $\alpha$ & $-0.8 \pm 0.1$ \\
\hline
\end{tabular}

from the empirical relation $r_{200}=0.813\left(k T_{\mathrm{B}} / \mathrm{keV}\right)^{0.5}(1+$ $z)^{-3 / 2} h_{70}^{-1} \mathrm{Mpc}$ (Navarro et al. 1995), which is slightly lower than $r_{200}=1.11 h_{70}^{-1} \mathrm{Mpc}$ using the global temperature and $r_{200}=1.05 h_{70}^{-1} \mathrm{Mpc}$ using the observed temperature distribution.

Furthermore, we apply an extended NFW model (e.g. Hernquist 1990; Zhao 1996; Moore et al. 1999),

$\rho_{\text {DM }}(r)=\delta_{\text {crit }} \rho_{\text {crit }}\left(\frac{r}{r_{\mathrm{s}}}\right)^{-\alpha}\left(1+\frac{r}{r_{\mathrm{s}}}\right)^{\alpha-3}$

to fit the data (Table 5) which provides a negative index. This indicates that the dark matter density has a flat core for CL $0024+17$.

We also present a comparison of the projected mass density and projected mass using the truncation radius of either $r_{200}=1.7 h_{70}^{-1} \mathrm{Mpc}$, predicted from the lensing data in Kneib et al. (2003) or a larger radius of $r=2.5 h_{70}^{-1} \mathrm{Mpc}$ (Fig. 11). The discrepancy is larger between the $\mathrm{X}$-ray and strong lensing measurements than between the X-ray and weak lensing measurements.

\subsection{Gas mass fraction}

The gas mass fraction distribution according to the definition $f_{\text {gas }}(r)=M_{\text {gas }}(r) / M(r)$ is shown in Fig. 12. We found $f_{\text {gas }}=$ $0.19 \pm 0.01 h_{70}^{-3 / 2}$ at $r_{200}=1.11 h_{70}^{-1} \mathrm{Mpc}$ using the global temperature and $0.20 \pm 0.03 h_{70}^{-3 / 2}$ at $r_{200}=1.05 h_{70}^{-1} \mathrm{Mpc}$ using the observed temperature profile, respectively. This result is comparable with the Chandra results $f_{\text {gas }}=0.14_{-0.02}^{+0.03} h_{70}^{-3 / 2}$ at $r_{200}=$ $1.40 h_{70}^{-1} \mathrm{Mpc}$ (Ota et al. 2004), and agrees with the WMAP measured baryon fraction of the Universe $f_{\mathrm{b}}=\Omega_{\mathrm{b}} / \Omega_{\mathrm{m}}=$ 0.166 , where $\Omega_{\mathrm{b}} h^{2}=0.0224$ and $\Omega_{\mathrm{m}} h^{2}=0.135$ (Spergel et al. 2003). We obtain a gas mass fraction of $0.11 \pm 0.01$ using the global temperature at $r_{2500}=0.30 \mathrm{Mpc}$ and $0.09 \pm 0.01$

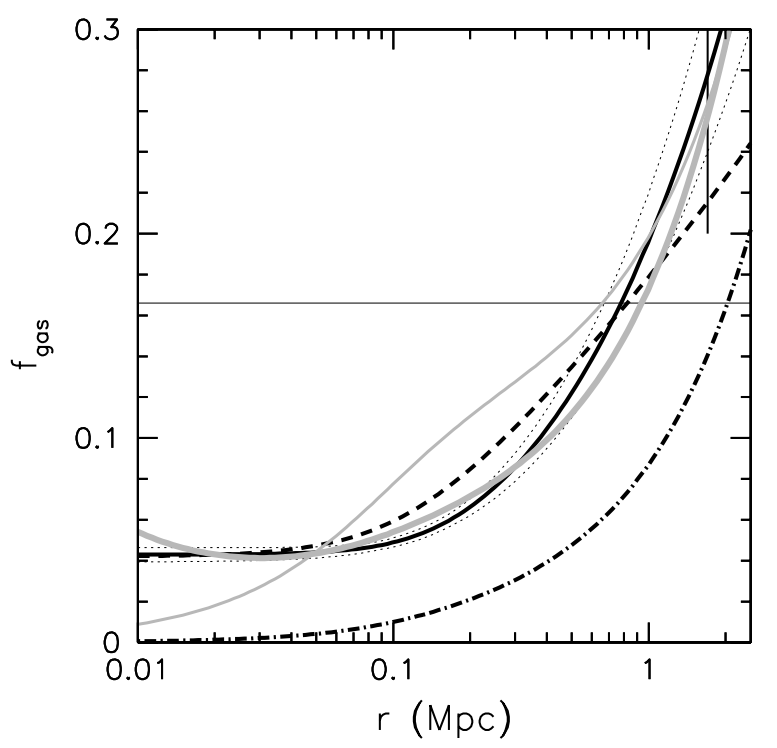

Fig. 12. Gas mass fraction as a function of radius. The lines have an identical meaning to those in Fig. 11 except that an additional horizontal line indicates the WMAP measurement $f_{\mathrm{b}}=\Omega_{\mathrm{b}} / \Omega_{\mathrm{m}}=0.166$ (Spergel et al. 2003).

Table 6. Measured parameters for CL 0024+17. Column (1): parameter; Cols. (2) and (3): values obtained: (i) in the $r<3^{\prime}$ region for line 2-4; (ii) using the global temperature and observed temperature distribution for line 5-7.

\begin{tabular}{lcc}
\hline \hline Parameter & \multicolumn{2}{c}{ Value } \\
\hline$k T_{\mathrm{B}}(\mathrm{keV})$ & \multicolumn{2}{c}{$3.52 \pm 0.17$} \\
$Z\left(Z_{\odot}\right)$ & $0.22 \pm 0.07$ \\
$L_{X}^{\text {bol }}\left(10^{44} h_{70}^{-2} \mathrm{erg} \mathrm{s}^{-1}\right)$ & \multicolumn{2}{c}{$2.9 \pm 0.1$} \\
$r_{200}\left(h_{70}^{-1} \mathrm{Mpc}\right)$ & 1.11 & 1.05 \\
$M_{200}\left(10^{14} h_{70}^{-1} M_{\odot}\right)$ & $2.3 \pm 0.1$ & $2.0 \pm 0.3$ \\
$f_{\text {gas }}\left(h_{70}^{-3 / 2}\right)$ & $0.19 \pm 0.01$ & $0.20 \pm 0.03$ \\
\hline
\end{tabular}

using the observed temperature profile at $r_{2500}=0.33 \mathrm{Mpc}$, which agrees with the measurements of Allen et al. (2002) based on Chandra observations of seven clusters yielding $f_{\text {gas }} \sim$ $0.105-0.138 h_{70}^{-3 / 2}$. However, this gas mass fraction at $r_{200}$ is slightly higher than the measurements of Sanderson et al. (2003) based on ASCA/GIS, ASCA/SIS and ROSAT/PSPC observations of 66 clusters yielding $f_{\text {gas }}=0.13 \pm 0.01 h_{70}^{-3 / 2}$, the measurements of Ettori et al. (2002) based on BeppoSAX observations of 22 nearby clusters, and the gas mass fraction for A1413 (Pratt \& Arnaud 2002) at $z=0.143$ based on XMM-Newton observations yielding $f_{\text {gas }} \sim 0.12 h_{70}^{-3 / 2}$.

The global parameters based on the XMM-Newton observations for CL $0024+17$ are given in Table 6 .

\section{Discussion}

\subsection{Metallicity}

We measure a global metallicity of $0.22 \pm 0.07 Z_{\odot}$ which is typical for a cluster with an insignificant cooling flow 
Table 7. Temperature and metallicity measurements in different bin size of the radius.

\begin{tabular}{llll}
\hline \hline Region & $k_{\mathrm{B}} T(\mathrm{keV})$ & $Z\left(Z_{\odot}\right)$ & $\chi^{2} /$ d.o.f. \\
\hline$r<0.40^{\prime}$ & $4.1 \pm 0.3$ & $0.16 \pm 0.10$ & $68.2 / 72$ \\
$r<0.50^{\prime}$ & $4.4 \pm 0.3$ & $0.10 \pm 0.09$ & $69.1 / 69$ \\
$r<1.00^{\prime}$ & $4.1 \pm 0.2$ & $0.25 \pm 0.08$ & $161.3 / 137$ \\
$r<1.25^{\prime}$ & $4.0 \pm 0.2$ & $0.23 \pm 0.07$ & $159.6 / 152$ \\
$r<1.50^{\prime}$ & $3.9 \pm 0.2$ & $0.23 \pm 0.07$ & $173.1 / 170$ \\
$r<1.67^{\prime}$ & $3.8 \pm 0.2$ & $0.24 \pm 0.07$ & $170.9 / 163$ \\
$r<2.00^{\prime}$ & $3.7 \pm 0.2$ & $0.23 \pm 0.07$ & $185.3 / 184$ \\
$r<3.00^{\prime}$ & $3.5 \pm 0.2$ & $0.22 \pm 0.07$ & $68.5 / 71$ \\
\hline
\end{tabular}

(Fabian \& Nulsen 1977). This metallicity value is in good agreement with the averaged metallicity $\langle Z\rangle=0.21_{-0.05}^{+0.10} Z_{\odot}$ for 18 distant clusters with redshift $0.3<z<1.3$ in Tozzi et al. (2003). However, Ota et al. (2004) obtain a much higher metallicity of $0.61-0.95 Z_{\odot}$. We use the images in the $5-6 \mathrm{keV}$ and $6-7 \mathrm{keV}$ bands as indicators of the iron $\mathrm{K}$ line and continuum emission, respectively, and obtain an iron to continuum map. We extracted the spectra from a suggested high metallicity region in the iron-to-continuum map at $\mathrm{RA}=00^{\mathrm{h}} 26^{\mathrm{m}} 32^{\mathrm{s}} .7$, Dec $=17^{\circ} 09^{\prime} 46^{\prime \prime} .3$ covering a radius of $0.32^{\prime}$. No significant high metallicity was obtained by either setting a free temperature $(5.4 \pm 1.6 \mathrm{keV})$ or setting the temperature to the emission weighted temperature $(4.09 \mathrm{keV})$ of the zone covering radii of $0.4<r<0.8^{\prime}$. For the same radius of $1.5^{\prime}$ as used by Ota et al. (2004), we measure a metallicity of $0.23 \pm 0.07$ and a temperature of $3.9 \pm 0.2 \mathrm{keV}$ (see Table 7) using the XMM-Newton data. Therefore the XMM-Newton data with higher significance provide no explanation for the high metallicity value obtained by Ota et al. (2004). The difference between XMM-Newton and Chandra metallicity measurements is an about 2 sigma effect, and may be due to the limited photon statistics in the Chandra data.

\subsection{Temperature gradient}

We derive an isothermal temperature of $\sim 3.9 \mathrm{keV}$ to a radius of $1.5^{\prime}\left(480 h_{70}^{-1} \mathrm{kpc}\right)$ and a power law model with an index of $\gamma=0.98$ outside this radius. The XMM-Newton temperature in the center is in good agreement with the Chandra measurement which extends to $1.5^{\prime}$ in the Chandra observation of CL 0024+17, covering the almost isothermal center $\left(r<1.3^{\prime}\right)$ in the XMM-Newton observations. We provide a more extended temperature profile based on the XMM-Newton observations in which the temperature profile decreases in the $1.3-3^{\prime}$ region. The strong temperature gradient at large radii makes the gravitational mass lower by a factor of $\sim 20-25 \%$ compared to the isothermal results. We found that the temperature gradient is not a sudden drop at a radius of $1.3^{\prime}$ by measuring the temperature in different bin sizes (see Table 7). Within a radius of $3^{\prime}$ for CL $0024+17$, we derived a polytropic index of 1.17 for the ICM in the outskirts which is lower than the adiabatic index of $5 / 3$. This implies that the outskirts are still convectively stable. We consider the uncertainty in the mass estimate caused by the temperature gradient by artificially increasing the slope parameter of the temperature distribution by a factor of $\sim 20 \%$, and find a decrease of the total mass by a factor of $\sim 10 \%$.

Since the soft band $(0.5-2 \mathrm{keV})$ is not as sensitive to the temperature map as the hard band $(2-7.5 \mathrm{keV})$, the HRM can be used as an indicator of the temperature distribution. Therefore the temperature map can be obtained from the HRM (e.g. Sanders \& Fabian 2001; Sanders et al. 2004; Akimoto et al. 2003). The $H R$ is also a reflection of absorption measure which affects the temperature (e.g. Akimoto et al. 2003). Thus we study the further properties of the features in the HRM by performing a spectral analysis. For the statistic reason, we extracted the spectra from one of the peaks suggesting high temperature in the HRM at RA $=00^{\mathrm{h}} 26^{\mathrm{m}} 30 \mathrm{~s} .9, \mathrm{Dec}=17^{\circ} 09^{\prime} 500^{\prime} 3$ covering a radius of $0.33^{\prime}$, and obtained a temperature of $5.8 \pm$ $1.0 \mathrm{keV}$ with metallcity fixed at $0.3 Z_{\odot}$ and $5.7 \pm 1.0 \mathrm{keV}$ with the metallicity free $\left(Z=0.88_{-0.58}^{+0.78} Z_{\odot}, L_{\mathrm{X}}^{\text {bol }}=0.43 \times\right.$ $10^{44} h_{70}^{-2} \mathrm{erg} \mathrm{s}^{-1}$ ). This supports the indication in the HRM of a high temperature region and not an artifact due to metallicity variations. However, the spectra can also be fitted by a power law with an index of $1.74 \pm 0.14$, which could then be attributed to some contamination from an AGN. This hot region is located exactly at the position of the substructure described in Czoske et al. $(2001,2002)$.

\subsection{Complex structure}

The Chandra image of the cluster core displays an indication of complex structure. The projection of the counts as a function of radius using the high spatial resulotion image from Chandra shows an asymmetric structure and a surface brightness "edge" in the central region. The galaxy alignment in the HST image is perpendicular to the "edge". Such an asymmetric central structure with an "edge" $\left(r<0.5^{\prime}\right)$ in the XMM-Newton HRM support the idea of a cluster merger.

\subsection{Comparison to lensing measurements}

The strongest disagreement between the X-ray mass profiles and the strong lensing results is found in the cluster center. The difference between the X-ray determined mass (Table 4) and the strong lensing mass of $1.59 \pm 0.04 \times 10^{14} h_{70}^{-1} M_{\odot}$ at the arc radius of $0.143 h_{70}^{-1} \mathrm{Mpc}$ (Broadhurst et al. 2000) and $2.37 \pm$ $0.36 \times 10^{14} h_{70}^{-1} M_{\odot}$ at the arc radius of $0.153 h_{70}^{-1} \mathrm{Mpc}$ (Tyson et al. 1998) is up to a factor of $\sim 4$.

This discrepancy is most probably due to the disturbed structure of the cluster center, highlighted by the elongated $\mathrm{X}$-ray iso-surface-brightness contours in the central region. The substructure found in the gravitational lensing mass distribution map by Kneib et al. (2003) is also supported by the $\mathrm{X}$-ray image. The velocity distribution of the galaxies can approximately be explained by a line-of-sight merger of two systems with a mass ratio of the order of 1:2 (Czoske et al. 2001, 2002). Therefore, the application of hydrostatic equilibrium to 
determine the mass in the central region of the cluster from the gas properties may not be valid.

We observed an elongation in the NW-SE direction in the HRM. The negative residual surface brightness in the cluster center demonstrates that the elongation flattens the surface brightness compared to an azimuthally symmetric structure. The XMM-Newton image analysis shows substructure to the NW of the cluster center which is marked by a galaxy (see Fig. 3) in Kneib et al. (2003). This galaxy shows significantly extended X-ray emission compared to the XMM-Newton PSF and has a luminosity of $L_{\mathrm{X}}^{\mathrm{bol}}=0.57 \pm 0.06 \times 10^{43} h_{70}^{-2} \mathrm{erg} \mathrm{s}^{-1}$ and a temperature of $0.87 \pm 0.13 \mathrm{keV}$ within $1^{\prime}$ at $\mathrm{RA}=$ $00^{\mathrm{h}} 26^{\mathrm{m}} 25^{\mathrm{s}} .8$, Dec $=17^{\circ} 12^{\prime} 03^{\prime \prime}$. 7 . Since this high surface brightness region does not have a lower temperature but probably rather has a higher temperature than its surroundings, it does not mark a "cold front" but a high pressure region which coincides with a mass concentration seen in the lensing map. This implies that the second mass peak is included within the gaseous halo of the cluster and not only seen projected onto the cluster.

\subsection{Comparison to velocity dispersion measurement}

The $M_{200}-\sigma_{\text {DM }}$ relation from simulations in Evrard \& Gioia (2002) gives a comparable velocity dispersion of $848_{-53}^{+51} \mathrm{~km} \mathrm{~s}^{-1}$ from the HST mass and a relatively low value of the velocity dispersion of $598_{-31}^{+29} \mathrm{~km} \mathrm{~s}^{-1}$ using the XMM-Newton mass. Girardi \& Mezzetti (2001) obtained a galaxy velocity dispersion of $911_{-107}^{+81} \mathrm{~km} \mathrm{~s}^{-1}$ for CL $0024+17$. The velocity dispersion derived from the XMM-Newton mass is in good agreement with the velocity dispersion measure in Czoske et al. (2002) who found velocity dispersions of $561_{-83}^{+95}$ and $554_{-304}^{+175}$ for the central component and foreground component which suggests a line-of-sight merger. If there is a line-of-sight merger, there are almost certainly additional galaxies in the line-of-sight which artificially increase the optical galaxy velocity dispersion if only one component is assumed.

\subsection{X-ray properties}

The mass estimates from the $M-T$ relations in Finoguenov et al. (2001) and in Bryan \& Norman (1998) are $M_{200}=5.96 \times$ $10^{14} h_{70}^{-1} M_{\odot}$ and $M_{200}=3.76 \times 10^{14} h_{70}^{-1} M_{\odot}$, which indicate a higher gravitational mass. We measure a bolometric X-ray luminosity of $2.9 \pm 0.1 \times 10^{44} h_{70}^{-2} \mathrm{erg} \mathrm{s}^{-1}$, which is higher than the luminosity of $1.26 \times 10^{44} h_{70}^{-2} \mathrm{erg} \mathrm{s}^{-1}$ obtained from the $L-T$ relation in Arnaud \& Evrard (1999) which might indicate a merger. The gas mass fraction at $r_{2500}$ for CL $0024+17$ agrees with the measurements of Allen et al. (2002) and Pratt $\&$ Arnaud (2002). The XMM-Newton measured gas mass fraction of $0.20 \pm 0.03 h_{70}^{-3 / 2}$ at $r_{200}$ for CL $0024+17$ is higher than the values in Sanderson et al. (2003), Ettori et al. (2002) and Pratt \& Arnaud (2002) for their cluster samples, which is possibly an indication for an underestimated mass beyond $r_{2500}$ as already noted from the $M-T$ relation. Thus we suggest that the merger leads to distortions on global scales which reduces the mass for CL 0024+17. However, on global scales the effect is smaller than in the cluster center. The relatively small distortion (less than a factor of 2) was found in several simulations (e.g. Evrard et al. 1996; Schindler 1996).

\subsection{Central cooling}

CL 0024+17 has a bright central galaxy and a compact lensing core. The entropy in the center is a little below the entropy floor value suggested by Lloyd-Davies et al. (2000). However, the cluster almost shows little evidence for a cooling flow or a central temperature decrease which should be found in most cooling flow clusters. This supports the scenario that the cluster center has been disturbed by a recent merger.

\section{Summary and conclusions}

We performed a detailed imaging and spectroscopic study of the XMM-Newton observations of the lensing cluster CL 0024+17, and obtained spatially resolved temperature, metallicity and density distributions. A temperature gradient was observed for the first time in CL 0024+17 at large radii $\left(1.3^{\prime}<r<3^{\prime}\right)$.

The image shows overlapping mass concentrations in the XMM-Newton X-ray data and HST optical data. The substructure which was observed by HST in Kneib et al. (2003) is confirmed in the X-ray data. The HRM shows an elongation in the NW-SE direction on a scale of 3.3' . Further spectroscopy confirms the temperature map suggesting high temperature region with $k_{\mathrm{B}} T=5.8 \pm 1.0 \mathrm{keV}$. Similar to Sersic $159-03$, the observed temperature distribution shows a temperature gradient at $1.3^{\prime}<r<3^{\prime}$. The modeling of the temperature distribution yields a polytropic index of 1.17 , lower than the adiabatic index of $\gamma=5 / 3$, which indicates a convectively stable state in the outskirts of CL $0024+17$.

The cluster does not show a pronounced cooling flow in spite of a dominant central galaxy. Therefore we suggest that a merger in the cluster center $\left(<0.1 h_{70}^{-1} \mathrm{Mpc}\right)$ has disrupted any previous cooling flow.

The mass estimate based on the precise measurements of the distributions of the temperature and gas density is lower by a factor of $\sim 20-25 \%$ than the mass obtained under the assumption of isothermality in the outskirts. The NFW model does not fit the X-ray derived mass profile very well in the center $\left(r<0.1 h_{70}^{-1} \mathrm{Mpc}\right)$. The extended NFW model fits the data but yields a negative index $\alpha$. We attribute this to the disturbance of the core.

The discrepancy remains between the X-ray gravitational mass and optical strong lensing mass at the radii of $0.143 h_{70}^{-1} \mathrm{Mpc}$ (Broadhurst et al. 2000) and $0.153 h_{70}^{-1} \mathrm{Mpc}$ (Tyson et al. 1998) by a factor of up to 4. The XMM-Newton results are marginally consistent within $2 \sigma$ using the global temperature with the weak lensing measurement in Kneib et al. (2003). CL 0024+17 is an example of a cluster for which weak lensing masses and X-ray masses are in acceptable agreement at large radii but for which the strong lensing masses disagree with the X-ray derived values at small radii (also e.g. A1689, Xue \& Wu 2002; Dye et al. 2001; Clowe \& Schneider 2001). 
The XMM-Newton measured parameters for CL 0024+17 deviate slightly from the empirical $M-T$ and $L-T$ relations. CL 0024+17 has a bright central galaxy and a compact lensing core but does not show a significant cooling flow. Similar to A2218 (Girardi et al. 1997; Pratt et al. 2004), the apparent discrepancy between the X-ray and gravitational lensing determined mass of CL $0024+17$ is most probably due to a lineof-sight merger of two almost comparable subsystems. In addition, filamentary structures could also contribute to the projected mass detected by lensing, but since this mass does not lie within the cluster core, it would not be included in the X-ray mass measurement. The line-of-sight orientation makes it difficult to reveal the merger structure in the X-ray data, but on the other hand it enhances the probability of finding strong lensing features. Thus, the application of the hydrostatic equilibrium assumption might break down in the central region. On global scales the agreement becomes better. The galaxy distribution, lensing mass maps, and X-ray data all provide a consistent description of the cluster morphology.

Acknowledgements. The XMM-Newton project is supported by the Bundesministerium für Bildung und Forschung, Deutschen Zentrum für Luft und Raumfahrt (BMBF/DLR), the Max-Planck Society and the Haidenhaim-Stiftung. We acknowledge Jacqueline Bergeron, PI of the XMM-Newton observation of the CDFS. Y.Y.Z. acknowledges receiving the International Max-Planck Research School Fellowship. W.F. acknowledges support from NASA Grant NAG5-9942 and the Smithsonian Institution. Y.Y.Z. thanks Alexey Vikhlinin, Gabriel Pratt, Kyoko Matsushita, Maxim Markevitch, Keith Arnaud, XiangZhong Zheng, Yas Hashimoto, Takaya Ohashi and Tae Furusho for useful discussions.

\section{References}

Akimoto, F., Kondou, K., Furuzawa, A., Tawara, Y., \& Yamashita, K. 2003, ApJ, 596, 170

Allen, S. W., Schmidt, R. W., \& Fabian, A. C. 2002, MNRAS, 334, L11

Anders, E., \& Grevesse, N. 1989, Geochim. Cosmochim. Acta, 53, 197

Arnaud, M., \& Evrard, A. E. 1999, MNRAS, 305, 631

Arnaud, M., \& Rothenflug, M. 1985, A\&AS, 60, 425

Arnaud, M., \& Raymond, J. 1992, ApJ, 398, 394

Böhringer, H., Soucail, G., Mellier, Y., Ikebe, Y., \& Schuecker, P. 2000, A\&A, 353, 124

Bonnet, H., Mellier, Y., \& Fort, B. 1994, ApJ, 427, L83

Broadhurst, T., Huang, X., Frye, B., \& Ellis, R. 2000, ApJ, 534, L15

Bryan, G. L., \& Norman, M. L. 1998, ApJ, 495, 80

Butcher, H., \& Oemler, A. 1978, ApJ, 219, 18

Cavaliere, A., \& Fusco-Femiano, R. 1976, A\&A, 49, 137

Clowe, D., \& Schneider, P. 2001, A\&A, 379, 384

Coia, D., McBreen, B., Metcalfe, et al. 2003, A\&A, submitted [arXiv: astro-ph/0310317]

Colley, W. N., Tyson, J. A., \& Turner, E. L. 1996, ApJ, 461, L83

Czoske, O., Kneib, J.-P., Soucail, G., et al. 2001, A\&A, 372, 391

Czoske, O., Moore, B., Kneib, J.-P., \& Soucail, G. 2002, A\&A, 386, 31
Dickey, J. M., \& Lockman, F. J. 1990, ARA\&A, 28, 215

Dressler, A., \& Gunn, J. E. 1982, ApJ, 263, 533

Dressler, A., Gunn, J. E., \& Schneider, D. P. 1985, ApJ, 294, 70

Dye, S., Taylor, A. N., Thommes, E. M., et al. 2001, MNRAS, 321, 685

Evrard, A. E., \& Gioia, I. M. 2002, Merging Processes in Galaxy Clusters, ed. L. Feretti, I. M. Gioia, \& G. Giovannini (Astrophysics and Space Science Library), 272, 253

Evrard, A. E., Metzler, C. A., \& Navarro, J. F. 1996, ApJ, 469, 494

Ettori, S., De Grandi, S., \& Molendi, S. 2002, A\&A, 391, 841

Fabian, A. C., \& Nulsen, P. E. J. 1977, MNRAS, 180, 479

Finoguenov, A., Reiprich, Th., \& Böhringer, H. 2001, A\&A, 368, 749 Ghizzardi, S. 2001, in-flight calibration of the PSF for the MOS1 and MOS2 cameras, EPIC-MCT-TN-011 (Internal report)

Girardi, M., Fadda, D., Escalera, E., et al. 1997, ApJ, 490, 56

Girardi, M., \& Mezzetti, M. 2001, ApJ, 548, 79

Gunn, J. E., \& Oke, J. B. 1975, ApJ, 195, 255

Hernquist, L. 1990, ApJ, 356, 359

Humason, M. L., \& Sandage, A., 1957, in Carnegie Yearbook 1956, Carnegie Institution of Washington, 61

Jones, C., \& Forman, W. 1984, ApJ, 276, 38

Kaastra, J. S. 1992, An X-Ray Spectral Code for Optically Thin Plasmas, Internal SRON-Leiden Report, updated version 2.0

Kaastra J. S., Ferrigno, C., Tamura, T., et al. 2001, A\&A, 365, L99

Kassiola, A., Kovner, I., \& Fort, B. 1992, ApJ, 400, 41

Kassiola, A., Kovner, I., Fort, B., \& Mellier, Y. 1994, ApJ, 429, L9

Kay, S. T. 2004, MNRAS, 347, L13

Kobayashi, C. 2004, MNRAS, 347, 740

Kneib, J.-P., Hudelot, P., Ellis, R. S., et al. 2003, ApJ, 598, 804

Koo, D. C. 1988, in Large-Scale Motions in the Universe, ed. V. G. Rubin, \& G. V. Gayne (Princeton Univ. Press), 513

Liedahl, D. A., Osterheld, A. L., \& Goldstein, W. H. 1995, ApJ, 438, L115

Lloyd-Davies, E. J., Ponman, T. J., \& Cannon, D. B. 2000, MNRAS, 315,689

Lumb, D. H., Warwick, R. S., Page, M., \& De Luca, A. 2002, A\&A, 389, 93

Makino, N., Sasaki, S., \& Suto, Y. 1998, ApJ, 497, 555

Markevitch, M. 1998, ApJ, 504, 27

Markevitch, M., Forman, W. R., Sarazin, C. L., \& Vikhlinin, A. 1998, ApJ, 503, 77

Mellier, Y., Fort, B., Soucail, G., Mathez, G., \& Cailloux, M. 1991, ApJ, 380, 334

Mewe, R., Gronenschild, E. H. B. M., \& van den Oord, G. H. J. 1985, A\&AS, 62, 197

Mewe, R., Lemen, J. R., \& van den Oord, G. H. J. 1986, A\&AS, 65, 511

Moore, B., Quinn, T., Governato, F., Stadel, J., \& Lake, G. 1999, MNRAS, 310, 1147

Navarro, J. F., Frenk, C. S., \& White, S. D. M. 1997, ApJ, 490, 493 (NFW)

Navarro, J. F., Frenk, C. S., \& White, S. D. M. 1995, MNRAS, 275, 720

Ota, N., Pointecouteau, E., Hattori, M., \& Mitsuda, K. 2004, ApJ, 601, 120

Ponman, T. J., Cannon, D. B., \& Navarro, J. F. 1999, Nature, 397, 135

Ponman, T. J., Sanderson, A. J. R., \& Finoguenov, A. 2003, MNRAS, 343,331

Pratt, G. W., \& Arnaud, M. 2002, A\&A, 394, 375

Pratt, G. W., Böhringer, H., \& Finoguenov, A. 2004, A\&A, submitted

Sanders, J. S., \& Fabian, A. C. 2001, MNRAS, 325, 178

Sanders, J. S., Fabian, A. C., Allen, S. W., \& Schmidt, R. W. 2004, MNRAS, 349, 952 
Sanderson, A. J. R., Ponman, T. J., Finoguenov, A., Lloyd-Davies, E. J., \& Markevitch, M. 2003, MNRAS, 340, 989

Schneider, D. P., Dressler, A., \& Gunn, J. E. 1986, AJ, 92, 523

Schindler, S. 1996, A\&A, 305, 756

Schuecker, P., Böhringer, H., Reiprich, Th., \& Feretti, L. 2001, A\&A, 378, 408

Shapiro, P. R., \& Iliev, I. T. 2000, ApJ, 542, L1

Smail, I., Ellis, R. S., Dressler, A., et al. 1997, ApJ, 479, 70

Soucail, G., Ota, N., Böhringer, H., Czoske, O., \& Mellier, Y. 2000, A\&A, 355, 433

Spergel, D. N., Verde, L., Peiris, H. V., et al. 2003, ApJS, 148, 175

Tozzi, P., Rosati, P., Ettori, S., et al. 2003, ApJ, 593, 705

Treu, T., Ellis, R.S., Kneib, J.-P., et al. 2003, ApJ, 591, 53
Tyson, J. A., Kochanski, G. P., \& Dell'Antonio, I. P. 1998, ApJ, 498, L107

Wallington, S., Kochanek, C. S., \& Koo, D. C. 1995, ApJ, 441, 58

Xu, H.-G., Jin, G.-X., \& Wu, X.-P. 2001, ApJ, 553, 78

Xue, S.-J., \& Wu, X.-P. 2002, ApJ, 576, 152

Zhao, H.-S. 1996, MNRAS, 278, 488

Zhang, Y.-Y., Finoguenov, A., Böhringer, H., et al. 2004a, A\&A, 413, 49

Zhang, Y.-Y., Finoguenov, A., Böhringer, H., et al. 2004b, Proc. Memorie della Societb Astronomica Italiana - Supplementi, in press [arXiv: astro-ph/0402533]

Zhang, Y.-Y., \& Wu, X.-P. 2003, ApJ, 583, 529 